\title{
Solar Intensity X-Ray and Particle Spectrometer SIXS: Instrument Design and First Results
}

\author{
J. Huovelin ${ }^{1}$ (D) R. Rainio ${ }^{2}$ E. Kilpua ${ }^{1}$ - A. Lehtolainen ${ }^{1}$ - S. Korpela ${ }^{1}$. E. Esko ${ }^{1}$. \\ K. Muinonen ${ }^{1}$ - E. Bunce ${ }^{3}$ - A. Martindale ${ }^{3}$ - M. Grande ${ }^{4}$ - H. Andersson ${ }^{5}$. \\ S. Nenonen ${ }^{5} \cdot$ J. Lehti $^{6}$ - W. Schmidt ${ }^{7}$ M. Genzer ${ }^{7}$ - T. Vihavainen ${ }^{8} \cdot$ J. Saari $^{6}$. \\ J. Peltonen ${ }^{6,2} \cdot$ E. Valtonen ${ }^{6} \cdot$ M. Talvioja ${ }^{9}$ P. Portin ${ }^{10} \cdot$ S. Narendranath ${ }^{11}$ \\ R. Jarvinen ${ }^{7,12}$ - T. Okada ${ }^{13}$ - A. Milillo ${ }^{14}$. M. Laurenza ${ }^{14}$ - E. Heino ${ }^{15,2}$ • P. Oleynik ${ }^{2}$
}

Received: 14 January 2020 / Accepted: 1 July 2020 / Published online: 14 July 2020

(C) The Author(s) 2020

\begin{abstract}
The Solar Intensity X-ray and particle Spectrometer (SIXS) on the BepiColombo Mercury Planetary Orbiter ("Bepi") measures the direct solar X-rays, energetic protons, and electrons that bombard, and interact with, the Hermean surface. The interactions result in $\mathrm{X}$-ray fluorescence and scattering, and particle induced X-ray emission (PIXE), i.e. "glow" of the surface in X-rays. Simultaneous monitoring of the incident and emitted radiation enables derivation of the abundances of some chemical elements and scattering properties of the outermost surface layer of the planet, and it may reveal other sources of X-ray emission,
\end{abstract}

The BepiColombo mission to Mercury

Edited by Johannes Benkhoff, Go Murakami and Ayako Matsuoka

J. Huovelin

juhani.huovelin@helsinki.fi

1 Department of Physics, University of Helsinki, Helsinki, Finland

2 Department of Physics and Astronomy, University of Turku, Turku, Finland

3 Space Research Centre, Department of Physics and Astronomy, University of Leicester, Leicester, UK

4 Institute of Mathematical and Physical Sciences, University of Wales, Aberystwyth, Wales, UK

5 Oxford Instruments Technologies Oy, Espoo, Finland

6 Aboa Space Research Oy, Turku, Finland

7 Finnish Meteorological Institute, Helsinki, Finland

8 Space Systems Finland Oy, Espoo, Finland

9 Talvioja Consulting Oy, Helsinki, Finland

10 Patria Aviation Oy, Tampere, Finland

11 UR Rao Satellite Centre, Indian Space Research Organisation, Bengaluru, India

12 Department of Electronics and Nanoengineering, School of Electrical Engineering, Aalto University, Espoo, Finland

13 Institute of Space and Astronautical Science, Japan Aerospace Exploration Agency, Tokyo, Japan

14 Institute for Space Astrophysics and Planetology, INAF, Roma, Italy

15 The University Centre in Svalbard (UNIS), Longyearbyen, Norway 
due to, for example, weak aurora-like phenomena in Mercury's exosphere. Mapping of the Hermean X-ray emission is the main task of the MIXS instrument onboard BepiColombo. SIXS data will also be used for investigations of the solar X-ray corona and solar energetic particles (SEP), both in the cruise phase and the passes of the Earth, Venus and Mercury before the arrival at Mercury's orbit, and the final science phase at Mercury's orbit. These observations provide the first-ever opportunity for in-situ measurements of the propagation of SEPs, their interactions with the interplanetary magnetic field, and space weather phenomena in multiple locations throughout the inner solar system far away from the Earth, and more extensively at Mercury's orbit.

In this paper we describe the scientific objectives, design and calibrations, operational principles, and scientific performance of the final SIXS instrument launched to the mission to planet Mercury onboard BepiColombo. We also provide the first analysis results of science observations with SIXS, that were made during the Near-Earth Commissioning Phase and early cruise phase operations in 2018-19, including the background X-ray sky observations and "first light" observations of the Sun with the SIXS X-ray detection system (SIXS-X), and in-situ energetic electron and proton observations with the SIXS Particle detection system (SIXS-P).

Keywords Planet Mercury · BepiColombo · Solar X-rays · Solar corona · Protons · Electrons · Instrumentation

\section{Introduction}

The Solar Intensity X-ray and particle Spectrometer (SIXS) onboard the BepiColombo MPO investigates the X-rays and energetic particles incident on the Hermean surface and in the inner heliosphere. In the inner solar system, they originate almost exclusively from the Sun, with small component coming from processes inside Mercury's magnetosphere. This radiation triggers observable X-ray emission when absorbed and scattered by the outermost surface layer of the planet. The X-ray emission from Mercury's surface is investigated by the MIXS (Mercury Imaging X-ray Spectrometer) instrument on BepiColombo (Fraser et al. 2010; Bunce et al. 2020). Thus, the SIXS-MIXS pair operates in principle similarly as commercially available X-ray Fluorescence (XRF) analyzers used in searching metal rich minerals for mining purposes on the Earth. Since the intensity and energy spectrum of both the $\mathrm{X}$-rays and the particles are highly variable and irregular on relatively small time-scales, simultaneity of measurements by SIXS and MIXS is a fundamental requirement in order to be able to quantitatively analyze the data from MIXS.

The main science objectives of SIXS are to:

- Monitor the Solar X-ray spectrum in the energy range 1 to $20 \mathrm{keV}$. This information is essential for the analysis of the X-ray spectrum observed by the Mercury Imaging X-ray Spectrometer (MIXS), to resolve the X-ray fluorescence and scattering on the surface of Mercury.

- Monitor the particle radiation environment to provide observational reference to the analysis of MIXS measurements of Particle Induced X-ray Emission (PIXE) on Mercury's surface.

- Monitor the energetic particle environment in the inner Solar System in order to better understand the propagation of electrons and protons in the inner heliosphere and space weather in the Earth's environment. 
- Observe the Sun continuously in soft X-rays with a high spectral resolution as a tool for understanding the hot solar corona and its contributions to solar physics and space weather.

The history of SIXS-like X-ray solar spectrometers dates back to the SMART-1 (Small Missions for Advanced Research in Technology) X-ray Solar Monitor (XSM), for which the design started in 1998. SMART-1 was the first European mission to the Moon. A review of SMART-1 XSM, the performance, data collected, and the first results is presented in Alha et al. (2008). Väänänen et al. (2009) made a study on cross-calibration of the XSM with the Geostationary Orbiting Environmental Satellites (GOES) and Reuven Ramaty High Energy Solar Spectroscopic Imager (RHESSI; Lin et al. 2002) solar X-ray instruments, which demonstrated the difficulty to match the results of high spectral resolution measurements made by XSM, which is nearly identical with SIXS X-ray detectors, and very low spectral resolution measurements of GOES and RHESSI. There was also another version of the XSM flown on-board the ISRO's Chandrayaan-1 lunar mission (Alha et al. 2009), which has resulted thus far in a number of scientific studies, e.g. the investigation of elemental abundances of the solar corona by Narendranath et al. (2014), and also very recently further studies reveal the changes in first ionization potential (FIP) bias during evolution of flares (Narendranath et al. 2020). This is enabled by the good spectral resolution and time resolution of these instruments. As a preparatory work for the fluorescence analysis, an experimental setup for the ground calibration of XSM (Huovelin et al. 2002) was utilized by Näränen et al. $(2008,2009)$ for studies of the planetary regolith effects on the X-ray fluorescence spectra. For the numerical studies of the regolith effects, see Parviainen et al. (2011), Bunce et al. (2020), and Rothery et al. (2020).

The particle detection system of SIXS (SIXS-P) relies on a traditional design principle of a detector stack of silicon detectors and a thallium doped cesium iodide scintillator with photodiode readout. The system employs the classical $\Delta \mathrm{E}-\mathrm{E}$ technique to measure the energy of a particle and to determine the species of incident electrons and ions. The same method was earlier applied by the team in the High Energy Detector (HED) of the Energetic and Relativistic Nuclei and Electron (ERNE) experiment (Torsti et al. 1995) on-board Solar and Heliospheric Observatory. Compared to ERNE / HED, SIXS-P is much more compact, consumes less power and has much wider field of view, being able to determine the particle flux in five orthogonal directions.

The SIXS and MIXS instruments share the same on-board computer and software (SW). The SW is designed so that they can be operated independently from each other. SIXS is able to make science observations also during the cruise phase, since the instrument has partial field-of-view to open space, thanks to its favourable position in the stack of BepiColombo spacecraft. Observations and monitoring of the dynamics of the high energy particles and $\mathrm{X}$-ray sky background in the interplanetary space is possible during the cruise phase and the swing-bys of the Earth, Venus and Mercury. It is also possible to make X-ray observations of the Sun with SIXS before the final Mercury science operations, which, however, requires a special $\mathrm{S} / \mathrm{C}$ attitude that is allowed only for very limited periods during the cruise phase. The long cruise phase will thus provide potential for SIXS to obtain highly valuable, and also new unique scientific measurements of energetic interplanetary electrons and protons in the inner solar system, and some opportunities for measurements of the X-ray Sun.

During the Mercury science phase, the main operational constraint of SIXS will be simultaneous operations with MIXS. Considering the scientific usefulness of SIXS data for its main purpose, all sources of radiation in the exosphere of the planet that change the Xray glow of Mercury in the energy range of MIXS are of significance, and determine the performance requirements of SIXS. The responsivity of Mercury's surface to the spectral 
distributions of various incoming forms of radiation are the fundamental factors that have constrained the types, the spectral ranges and resolutions, the dynamical ranges, and aperture sizes of detectors of the SIXS instrument.

X-rays emitted from the Sun travel with a practically direct path to the dayside surface of Mercury. The paths and energies of particles, on the other hand, depend on the solar magnetic field and are affected by the magnetosphere of Mercury. Thus, the relations between particles observed by BepiColombo and those hitting the surface of Mercury below the S/C are complicated, and depend on the orbital phase and position of BepiColombo in the Hermean magnetosphere. These phenomenological aspects determine the required angular resolutions for the detectors of the SIXS instrument. Since we know the direction of the incoming X-rays we do not need any angular resolution for the X-ray detectors. However, we need some degree of angular resolution for the particle measurements to be able to separate with SIXS the components of particle fluxes that will hit Mercury's surface in the footprint of MIXS images.

In order to be able to utilize the MIXS measurements at any orbital phase of BepiColombo MPO around Mercury, SIXS is required to be able to make relevant measurements throughout the orbit as well. Thus, on the dayside of Mercury, an X-ray detector of the SIXS instrument must have a direct view towards the Sun, and the particle detector is required to be able to determine the particle fluxes and directions at any orbital phase, including the night side of Mercury. These requirements are translated to a requirement for the field-ofview for the X-ray and particle detectors of SIXS, and are the basis for the optical design of the X-ray and particle measurement systems of SIXS.

SIXS data is not only useful for MIXS and independent science with SIXS, but SIXS can also contribute to investigate the effects of Sun's major events on the planetary magnetosphere and exosphere jointly with other BepiColombo instruments devoted to the Mercury's environment, like the SERENA (Orsini et al. 2020), MERMAG (Heyner et al. 2020), PHEBUS (Quémerais et al. 2020) instruments onboard the Mercury Planetary Orbiter (MPO, "Bepi"), and the Mercury Magnetospheric Orbiter (MMO, "Mio") instruments. Furthermore, major solar energetic particle (SEP) events could produce electronic disturbances and spurious signals on some particle detectors, thus SIXS, together with the BERM radiation monitor of the MPO (Moissl et al. 2020), can support the analysis of SERENA (Orsini et al. 2020) observations, and also observations by the instruments of the MMO (Saito et al. 2020). The studies of the temporal and spectral variability of the solar X-ray corona, the temporal behaviour and X-ray spectral classification of solar flares, and the temporal and spectral variability of proton and electron radiation near Mercury with SIXS have connections to the observations with the above instruments. These will together lead to better understanding the observed physical processes in the Hermean magnetosphere.

The technology developed for the X-ray detector system of SIXS has also been developed in another direction by industry for operational use in future space weather observation platforms. ESA has planned to host an X-ray Flux Monitor (XFM) developed by largely the same industrial consortium that built SIXS on-board the next space weather mission to Lagrange point 5 in ESA's Space Safety Programme (S2P). The mission may be launched before the end of BepiColombo mission. There is also a preceding Low Earth Orbit (LEO) demonstration of XFM planned for launch in 2021 onboard a $2 \mathrm{U}$ CubeSat, that will measure the solar X-ray spectra with approximately the same resolution as SIXS, built in ESA's General Support Technology Programme (GSTP). The maximum lifetime of the LEO demonstration mission called Sunstorm 1 is 2 years. The particle detection concept developed for SIXS has been further developed for radiation monitoring in the Radiation Monitor (RADMON) experiment (Gieseler et al. 2019; Oleynik et al. 2019) aboard Aalto-1, a 3U CubeSat in polar LEO launched in June 2017. 
The first comprehensive description of the SIXS instrument, as well as its scientific and technological background and heritage and science plan is published in Huovelin et al. (2010), and the present paper is an updated description, including the final design, performance and first results after the launch of BepiColombo.

\section{Scientific Objectives}

The science objectives of SIXS can be divided to those related to X-ray measurements (SIXS-X) and those related to energetic particles measurements (SIXS-P). Further division of science objectives can be made regarding whether science is done in cooperation with MIXS (or other BepiColombo instruments) or independently with only SIXS.

\subsection{X-Ray Measurements}

The main scientific objective regarding SIXS's X-ray measurements is to enable a valid analysis of the X-ray fluorescence and scattering on the surface of Mercury based on the observations by MIXS. The fluorescence and scattering results from direct X-rays from the Sun on the dayside of Mercury's surface including dawn and dusk regimes. SIXS will measure the solar X-ray spectrum and is a crucial input for interpretation of MIXS spectra.

To meet this science objective, several requirements are imposed on SIXS to provide sufficiently accurate information on the physical X-ray spectrum incident on Mercury's surface. MIXS covers the emission spectrum in the energy range $0.7-10 \mathrm{keV}$. Considering this, the sufficient energy range for SIXS is $1-20 \mathrm{keV}$ and energy resolution of roughly $400 \mathrm{eV}$ at $6 \mathrm{keV}$. As the energy resolution of the semiconductor diode X-ray detector degrades under a long time of exposure to space radiation, the BOL (Beginning of Life) energy resolution has to be significantly better than the above value.

The key independent science objective regarding SIXS X-ray measurements is to investigate the variations in the intensity and spectrum of solar X-rays. This enables studies of solar corona and in particular of solar flares, and physical processes giving rise to the X-ray emission of the Sun. The independent science will include statistical studies of the flare spectra, in order to better understand the relation of the emission measure and temperature with the physical dimensions and dynamical properties of individual flares. This aim motivates studies of the connections between the spectra and flare morphology by combining the observations of SIXS with those of Solar Orbiter, Solar Dynamical Observatory, and also the X-ray monitoring data of the Sun by the operational Space Weather satellites.

Also, important are studies on FIP bias in the corona (e.g., Laming 2015). Recent studies show that the FIP bias changes during flares and it is connected to the magnetic field topology in the pre-eruptive structure (Narendranath et al. 2020). Disk averaged coronal abundances measured with SIXS-X can also be compared to those measured in stars. These studies can give clues as to the physical connection of the X-ray emission with solar energetic particles (SEPs), and the relation of solar X-ray emission and flares with Coronal Mass Ejections (CMEs) and generation mechanisms of flares and CMEs. The proximity of the Sun enables also higher time resolution and sensitivity for lower X-ray emission levels and thus weaker flares. These may lead to new findings connected with micro- and nanoflares, the debated role of nanoflares to coronal heating, and the origin of the ubiquitous low intensity $\mathrm{X}$-ray emission of the Sun.

Mercury's perihelion distance is 0.307 AU, which means that solar X-ray fluxes and spectra measured at $1 \mathrm{AU}$ must be rescaled by a factor 10.6 at maximum to obtain realistic 
requirements for the upper limit of the sensitivity range of SIXS X-ray detectors. For aphelion distance of $0.47 \mathrm{AU}$, the expected fluxes are approximately 4.5 times higher than those at $1 \mathrm{AU}$. An X1 flare at Mercury will thus produce a physical flux $10.6 \times 10^{-4} \mathrm{~W} / \mathrm{m}^{2}$ at maximum and $4.5 \times 10^{-4} \mathrm{~W} / \mathrm{m}^{2}$ at minimum. The intensities of all other flare levels scale similarly, all without any difference in spectrum shape from that observed at Earth distance. The range of expected spectra and intensities can thus be derived with high accuracy, since extended data from previous missions with suitable spectrum range and spectral resolution are available.

There is also a very extended data of X-ray fluxes in range $1-8 \AA$ (1.55-12.4 keV) from the Geostationary Operational Environmental Satellite (GOES) X-ray flux monitoring, which enables a way to predict the range of X-ray emission levels and probabilities of the occurrence of various flares on the Sun. A good basis for predicting the occurrence rate of flares of various magnitudes is the automated statistical analysis of solar X-ray fluxes over 37 years provided by Aschwanden and Freeland (2012). This analysis yields the best available independent estimate of the expected occurrence rate of various flares based on $\mathrm{X}$-ray data in a relevant energy range.

Table 1 shows predictions based on this data source for solar X-ray emission during a relevant time period for the science operations of the BepiColombo mission. These data enable optimisation of the dynamical range of SIXS X-ray detector sensitivity to best suit the conditions during the BepiColombo mission. To further enhance the dynamic range, one of the SIXS X-ray detectors has an aperture that is $50 \%$ bigger in diameter than the other two, and thus the aperture area is 2.25 times bigger, which extends the dynamic range of SIXS at the low end and enables better $\mathrm{S} / \mathrm{N}$ for weak flares. The increased sensitivity may cause signal saturation and pile-up effect in the spectrum during periods when the bigger detector makes measurements during strong (i.e. X-level) flares. However, since the statistical probability of the occurrence of very strong flares is very small, the sensitivity advantage during periods of lower flux more than compensates this disadvantage, which can be seen in Table 1. Aschwanden and Freeland (2012) found the following general statistical relationship: The number of flares during a defined period is inversely proportional to the peak intensity of flares, e.g., M flares occur, on average, 10 times more often than X flares. There may be interesting physical implications from this finding related to e.g. the total average energy release rate of the solar corona, which deserves attention in the scientific analysis of the future X-ray (and also particle) data of SIXS.

\subsection{Particle Measurements}

A component of X-ray fluorescence will also result from energetic particles incident on the Mercury's surface (e.g. Starr et al. 2012). This includes both particles accelerated by the processes at the Sun, and those captured and accelerated in the magnetosphere of Mercury. To get a comprehensive description of the X-ray-inducing radiation components in the Hermean environment, the SIXS experiment needs to perform measurements of fluxes and rough spectra of energetic particles incident on the planet's surface. Since particle radiation incident on Mercury's surface can occur on the night side as well as day side surface, particle measurements are necessary throughout the MPO orbit. The most abundant species producing particle induced X-ray emission (PIXE) are protons and electrons. Harjunmaa (2004) showed that large energetic proton events are able to produce a fluorescence yield comparable to an X-ray flare. The effect of PIXE is most pronounced for the lines with largest X-ray energies.

The X-ray yield of the target material varies as a function of incident particle and its kinetic energy. The cross section of PIXE is determined by the $\mathrm{K}$ - and L-shell ionisation 
Table 1 Predictions for the number of various solar flares in 2025 and 2027 based on Aschwanden and Freeland (2012). The flare intensities are at Earth distance, and need to be rescaled for BepiColombo, using the actual distance of the MPO S/C from the Sun. The general flare classification $(\mathrm{X}, \mathrm{M}, \mathrm{C})$ is used

Corresponding to 2025 (arrival at Mercury orbit)

\begin{tabular}{|c|c|c|c|c|c|}
\hline Year & 2003 & 1992 & 1981 & Average & Standard deviation \\
\hline All Flares & 12283 & 12488 & 15527 & 13433 & 1817 \\
\hline$X 1-9$ & & & & 12 & 2 \\
\hline M1-9 & & & & 121 & 16 \\
\hline C1-9 & & & & 1209 & 164 \\
\hline$<\mathrm{C}$ & & & & 12091 & 1635 \\
\hline \multicolumn{6}{|c|}{ Corresponding to 2027 (end of mission) } \\
\hline Year & 2005 & 1994 & 1983 & & \\
\hline All flares & 6706 & 4844 & 9566 & 7039 & 2379 \\
\hline $\mathrm{X} 1-9$ & & & & 6 & 2 \\
\hline M1-9 & & & & 63 & 21 \\
\hline C1-9 & & & & 634 & 214 \\
\hline$<\mathrm{C}$ & & & & 6335 & 2141 \\
\hline
\end{tabular}

cross sections. Almost all collisions lead to ionization and only a small fraction lead to excitation. The kinetic energy of maximum cross-section for $\mathrm{K}$ - and $\mathrm{L}$-shell ionisation is approximately proportional to the binding energy $B$ of the shell. For electrons, the peak of the ionisation cross section is at about three times the binding energy (Santos et al. 2003), giving the electron energy for maximal K-shell ionisation in the region of 2-20 keV for the target elements that are of scientific interest for MIXS. These are the elements with lines between 0.7 and $7.5 \mathrm{keV}$. In addition, especially for heavy target elements, there is a long tail of high $\mathrm{K}$-shell ionisation cross section extending to relativistic electron energies. For protons, the peak in the K-shell ionisation cross sections occurs at much higher energies, i.e., at $\left(m_{\mathrm{p}} / m_{\mathrm{e}}\right) B$ (Sera et al. 1980), yielding $\sim 1.4-15 \mathrm{MeV}$ for the elements of interest. In the energy range beyond the peak, the cross-section decrease follows a power-law in energy (e.g., Harjunmaa 2004). The ionisation cross-section peak values are from a few tens to tens of thousands of barns. The X-ray production cross section is a target-materialdependent fraction of the ionisation cross section. The coefficient of proportionality, i.e., the fluorescence yield, is between a few and a few tens of percent for the elements of interest. In addition to the fluorescence cross-section, we need information on the energy spectra and relative abundances of protons and electrons to assess their relative contribution to PIXE.

The resources of the SIXS experiment only allow one particle measurement technique to be employed, rendering the measurement of the entire energy range for electrons and protons a very difficult, if not impossible task. The trade-off has been made in favour of protons and high-energy electrons, because it is possible to use the MIXS X-ray measurements of the bremsstrahlung continuum caused by multi-keV electrons hitting the surface of Mercury to estimate the electron intensities below a few tens of keV (Huovelin et al. 2010). A further reason in favour of optimising the measurements for the protons and high-energy electrons is that even if it would be possible to measure the low-energy electron fluxes locally, there would be a less direct connection between the measured fluxes at MPO and those hitting the planetary surface below the spacecraft. This is because non-relativistic electrons 
are tightly bound to the magnetic field lines of the magnetosphere and the field lines are typically not vertical. The Larmor motion of $\mathrm{MeV}$-protons can bring them from the day-side magnetopause to the planetary surface across the field lines, but electrons follow the field lines up to very high energies. Also, the MMO electron measurements (extending to some $100 \mathrm{keV}$ ) will give information on the electron fluxes in the system and be useful for the purpose of interpreting MIXS observations.

In addition to its contribution to the fluorescence analysis, the SIXS particle spectrometer will provide very valuable data on SEP fluxes close to the Sun. The advantage of the BepiColombo mission is that it will stay close to the Sun all the time, which will eliminate much of the effects of interplanetary transport and allow a more direct study of solar particle accelerators than measurements at $1 \mathrm{AU}$. A two-year mission (including the foreseen extension period) close to the solar maximum will provide valuable information on energetic particle transport in the inner heliosphere and on the acceleration mechanisms of SEPs in solar flares and at coronal and interplanetary shocks. The seven-year cruise phase in the ascending phase of cycle 25 will also add to that goal.

\section{Instrument Description}

\subsection{Technical Specifications}

The SIXS instrument sensor unit consists of an X-ray detection system, a particle detection system, instrument electronics, housing, and a thermomechanical structure, including a heat shield and a radiator that cover the instrument housing, except the particle detector system and aperture holes of the three X-ray detectors. The backend electronics, including the data processing unit, the power supply, and the telemetry hardware is inside the common unit with MIXS, called the MIXS Electronics Box (MEB), that is connected with SIXS with cables routed via the bottom of the SIXS sensor unit to the MEB, which is inside the MPO housing. The SIXS sensor unit itself is located at the corner of the MPO radiator side in such a way, that the SIXS-X visibility to the Sun and the SIXS-P visibility to the full sky is good during all the Mercury orbit phases. There is partial sky visibility for SIXS even during the cruise phase, although part of the sky is obstructed by the sunshield of the MMO spacecraft.

Because of the need to always be in the line-of-sight of the Sun, the sensor unit thermomechanical design is quite complex. The need for three X-ray detectors is a result of a finite field of view combined with the way the MPO attitude (with respect to the Sun) changes during the Mercury year and the fact that SIXS cannot rotate on the spacecraft. In order to provide a line-of-sight to the Sun at all times at the day side of Mercury, SIXS X-ray detector system consists of three X-ray detectors pointed at different directions. The arrangement is shown in Fig. 1. The design is mostly driven by the harsh thermal environment but should at the same time give optimized view factors for the sensors and provide electromagnetic and radiation shielding for the electronics. It will have to withstand the intense solar thermal radiation at Mercury distance from the Sun, which means that the worst-case solar heat load is more than an order of magnitude higher than for an instrument deployed around the earth. In addition, the Mercury surface with a temperature above $400{ }^{\circ} \mathrm{C}$ at the sunlit side is visible to the instrument and presents a heat load, both in terms of direct infrared and UV-visible albedo radiation, almost of the same magnitude as the direct solar radiation. Figure 1 shows the high- level sensor unit design visualized in a detached arrangement with a heat shield facing the sun and a radiator facing the planet and cold space. 


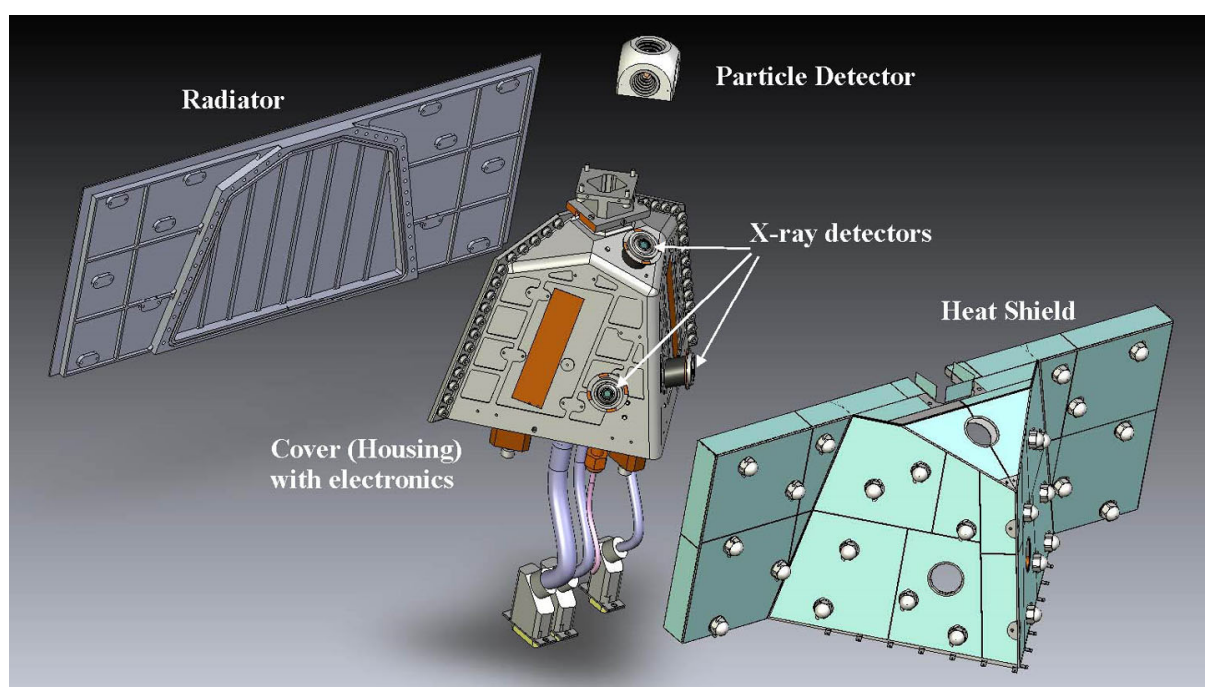

Fig. 1 SIXS sensor unit, particle detector package, and surface elements detached

The heat shield is a multi-layer structure with the outermost part a complex shaped titanium sheet with custom cut tiles of optical solar reflectors (OSR) bonded to it using an electrically conductive silicone. The OSRs which are facing the direct sunlight has a very low solar absorbance in the UV-visible wavelengths, in order to reject a major part of the solar heat load. They also have a high infrared emittance to allow heat to be re-emitted to cold space since the sun is only covering a small fraction of the total OSR view-factor. All other parts in the direct sun-light are either polished aluminium, material covered by vapour deposited aluminium or as in the case of the particle detector collimator, manufactured by single-point diamond turning to give the part a very low surface roughness $(\mathrm{Ra}<5 \mathrm{~nm})$ which in turn results in a high reflectivity in UV-visible wavelengths. Behind the outermost OSR-covered titanium sheet is an intermediate aluminium sheet with a similar shape, followed by a 20-layer conventional multi-layer insulation (MLI) blanket. All these layers are held in place by low conductivity polymer spacers which are locked at the end of the stack with cap nuts. This multi-layer structure allows a controlled amount of heat to flow into the sensor unit, without the risk of overheating the outermost OSR layer. The final construction of SIXS is shown in laboratory in Fig. 2.

The position of the SIXS instrument on the MPO platform is challenging; the area intended to offer cooling for the instrument sees the very high temperature Mercury's surface most of the time at orbit. To avoid the heating effect of the strong IR and albedo fluxes from the planet surface and yet to be able to offer cooling for SIXS, the radiator constructed from aluminium has saw-tooth shaped grooves machined into it. The sides of the grooves seeing mostly cold space are coated with a white high emissivity coating enabling heat dissipation from the unit. The sides seeing mostly the Mercury's surface are machined by single-point diamond turning to give them a very high reflectivity against the planet's heat load. The radiator is thermally well coupled to the housing, and on surfaces exposed to solar radiation are insulated in the same manner as the main instrument body: OSR outer surface, MLI insulation, and attachment system that promotes heat transfer by thermal radiation only. The radiator is shown in Fig. 3. 
Fig. 2 SIXS sensor unit during final mounting of the heat shield. The view is from the solar side with the array of OSR tiles, heat shield locking nuts, the windows of the three X-ray detectors and the particle detector collimator visible
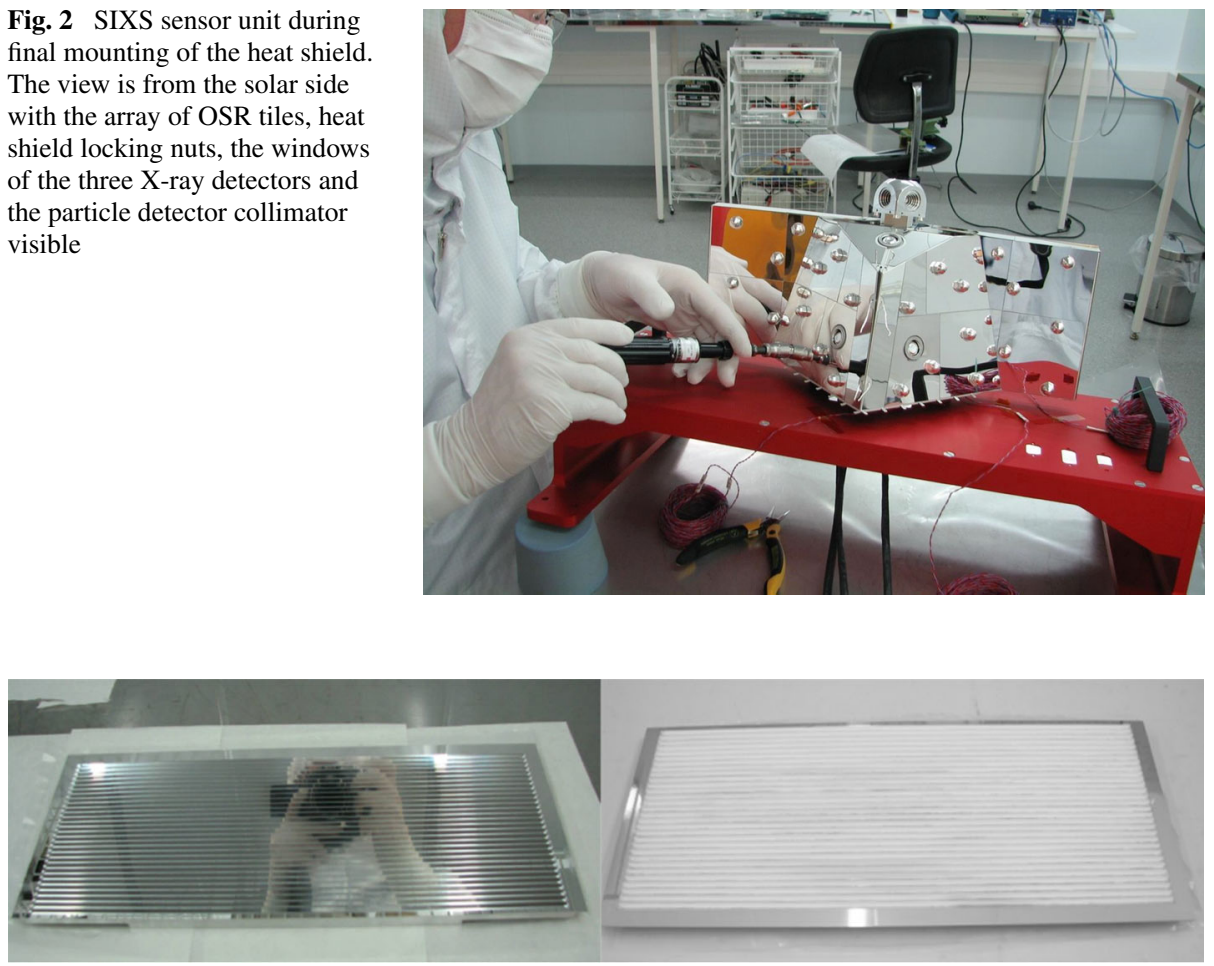

Fig. 3 Radiator seen from two viewing directions, the surface facing up towards the cold sky (right) and highly reflecting surface down towards the planet (left)

It shall be noted that the MPO spacecraft presents no thermal protection for SIXS, except at the bottom where it has an interface with the spacecraft multi-layer insulation. SIXS is also thermally decoupled from the spacecraft by means of three thermal spacers, made of a low heat conductance polymer. As can be seen in Fig. 4 the unit is mounted on a bracket with its radiator in-line with the spacecraft radiator to minimize radiative heat load from the spacecraft and optimize the view factor to cold space. Because of the high need for passive cooling of the unit, it will run at very cold temperature during certain times of the mission and has therefore an electrical, spacecraft-controlled safety heater installed. SIXS was qualified in the temperature range $-65^{\circ} \mathrm{C}$ to $+70^{\circ} \mathrm{C}$ for the housing, electronics and sensors, and $-115^{\circ} \mathrm{C}$ to $+300^{\circ} \mathrm{C}$ for the outer layer of the heat shield.

Figure 5 shows a cross section of the sensor unit housing revealing the positions of the $\mathrm{X}$-ray and particle detector subsystem parts. Electrically these are two separate subsystems, each of which are described in more detail below. However, it should be noted that due to their physical placements close to each other and the low noise level of the detectors involved, it was of utmost importance to electromagnetically shield the two systems from each other. This was done by means of completely enclosing the particle detector mainboard with copper coated polymer film connected to electrical ground. The interface of these two subsystems to the SIXS on-board computer and power supply unit was by a harness consisting of two separate cables, one for power lines and one for data lines. The data interface was in both cases a custom serial link using LVDS signaling. 


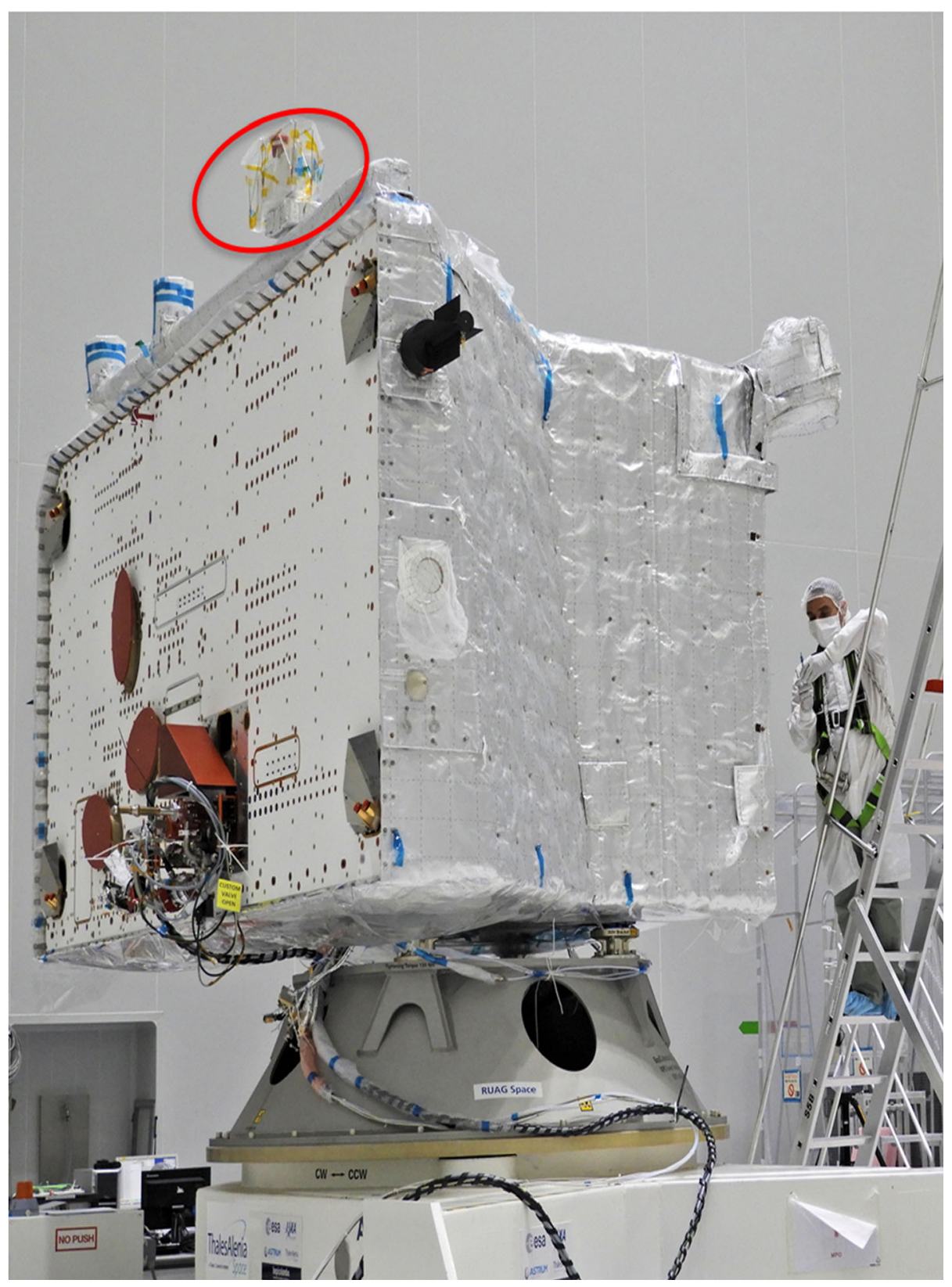

Fig. 4 SIXS sensor unit (in red circle) on the MPO spacecraft view from the radiator side. Photo courtesy of European Space Agency, ESA

\subsection{X-Ray Detection System}

\subsubsection{Description}

In order to provide a line-of-sight to the Sun at all times at the day side of Mercury, SIXS $\mathrm{X}$-ray detector system consists of three X-ray detectors pointed at different directions. The 
Fig. 5 Cross section of the SIXS sensor unit

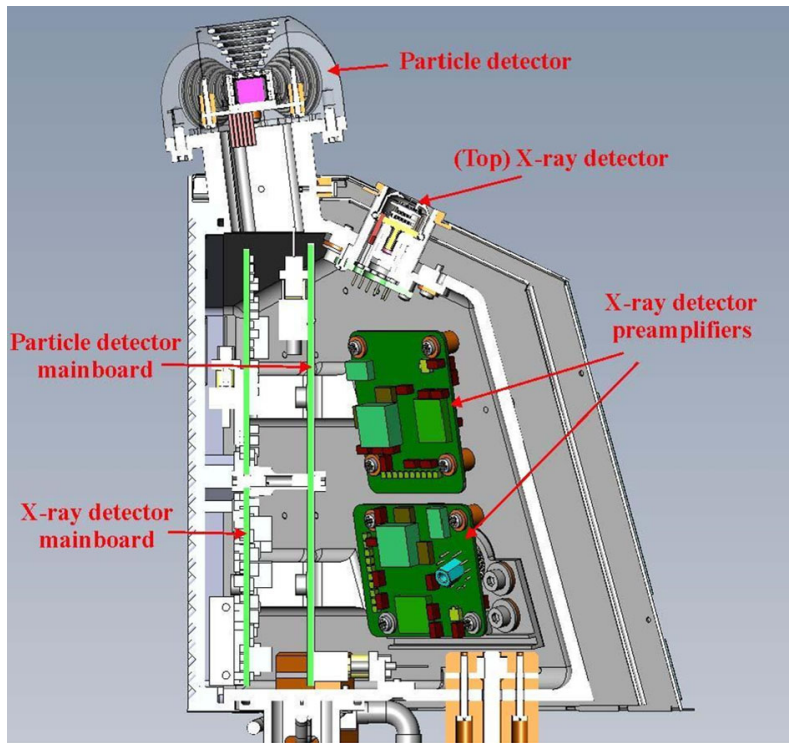

arrangement is shown in Fig. 1. The three X-ray detectors, that measure X-rays in the range 1 to $20 \mathrm{keV}$, are based on $0.3 \mathrm{~mm}$ thick crystals of Si PIN diodes and cooled by means of a thermo-electric (Peltier) cooler. An NTC thermistor measures the actual temperature of each PIN diode. An aperture, made from $125 \mu \mathrm{m}$ thick Au foil, defining a precise active X-ray measuring area for the PIN diode, is placed on the front surface of the diode. The aperture is $0.2 \mathrm{~mm}$ in diameter for two of the detectors and $0.3 \mathrm{~mm}$ for the third, giving active areas of roughly $0.03 \mathrm{~mm}^{2}$ and $0.07 \mathrm{~mm}^{2}$, respectively. However, as explained in detail below, the PIN diode sizes are significantly larger in order to also allow detection of Xrays from the specially developed in-flight calibration source (Lehtolainen et al. 2011). All these components are hermetically sealed behind a $50 \mu \mathrm{m}$ thick Be window into a transistor outline type package as shown in Fig. 6. The main function of the Be window is to reduce the count rate at low X-ray energies in order to prevent saturation of the detector during strong X-ray events. The effect of the window on the low energy cut-off in transmission is visualized in Fig. 12, where the decrease of the quantum efficiency towards low energies is dominated by the absorption in the Be window. The edge of the X-ray entrance hole in the steel cup has a $10 \mu \mathrm{m}$ thick Au layer plated to it to avoid fluorescent X-rays from the cup material to be detected.

The detectors have an internal calibration source in the aperture window structure, as shown in Fig. 6. Fe55 has a half-life of 2.737 years and it emits $\mathrm{Mn} \mathrm{K} \alpha$ and $\mathrm{K} \beta \mathrm{X}$-rays at approximate energies 5.9 and $6.5 \mathrm{keV}$. The activity of the Fe 55 sources was $870 \mathrm{MBq}$ at the time of purchase in 2011. This was chosen so that given the 2.737 year half life of Fe55, the intensity of the calibration signal will be sufficiently high would upon arrival to Mercury, with sufficient margin in the case of foreseen possible delay in the launch of BepiColombo. The estimated calibration signal upon arrival to Mercury's orbit in the end of 2025 will be $\sim 32$ cps, which is sufficient for the determination of the energy scale and resolution of the spectrum.

The source was manufactured by electroplating Fe55 isotope material onto a gold substrate. Together with a $10 \mu \mathrm{m}$ thick Ti foil, yielding additional $\mathrm{Ti} \mathrm{K} \alpha$ and $\mathrm{K} \beta$ fluorescence lines at 4.5 and $4.9 \mathrm{keV}$, they were placed on top of the aperture, which also have laser 

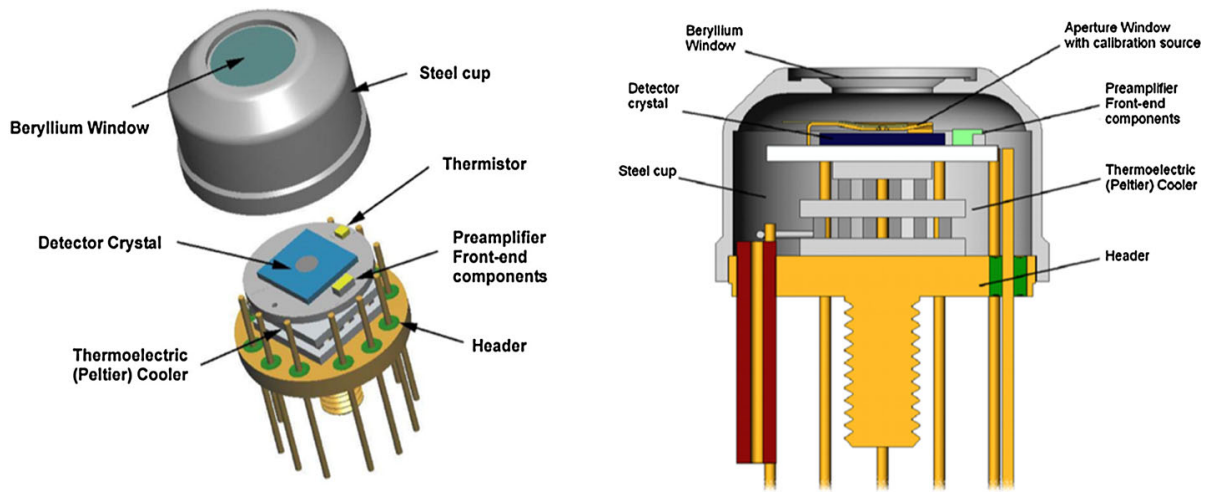

Fig. 6 SIXS X-ray detector package (one detector) exploded view (left) and cross section (right)
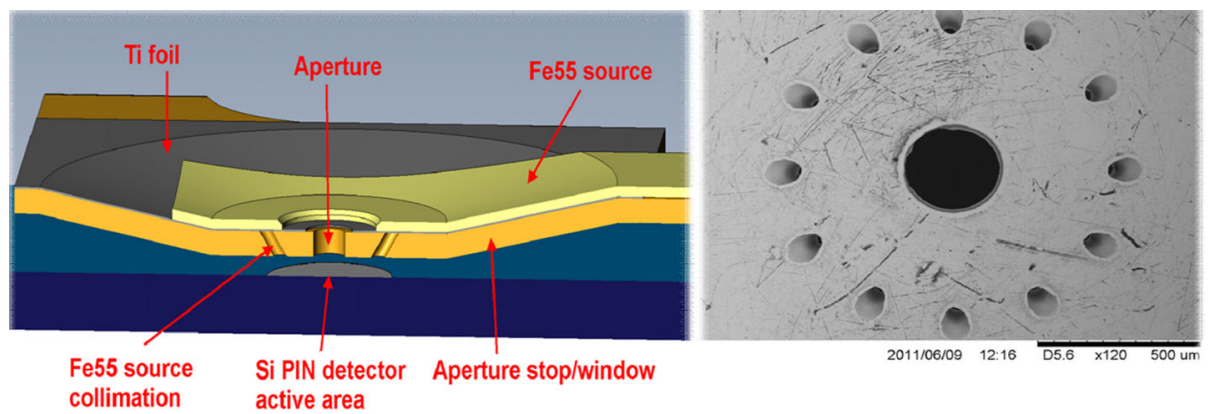

Fig. 7 Close-up and cross section of SIXS X-ray detector aperture and calibration source assembly (left) and scanning electron microscope image of one of the apertures (right)

drilled holes to collimate the source radiation onto the Si PIN diode without interfering with the field of view towards the sun as shown in Fig. 7. The figure also shows a scanning electron microscope image of an actual aperture stop with the larger hole in the middle acting as the aperture for solar X-rays and the smaller holes surrounding it acting as collimators for the calibration source. The smaller holes ( $70 \mu \mathrm{m}$ diameter) were drilled tilted at a $35^{\circ}$ angle from the normal of the foil, in order to minimize the needed active area of the Si PIN diode, which was $0.5 \mathrm{~mm}^{2}$ for the smaller aperture and $0.95 \mathrm{~mm}^{2}$ for the larger aperture.

The X-ray detector subsystem naturally also includes a complete spectroscopic chain of amplifiers, analog-to-digital (ADC) converter, Peltier controller, high voltage supply and all control functions. As mentioned in Sect. 3.2, the preamplifiers are situated on separate printed circuit boards (PCB) close to the detector packages. These are interconnected by cables to the rest of the electronics situated on a larger main board. The three X-ray detectors are multiplexed and only one detector is operational at a time.

The preamplifiers are based on a charge sensitive amplifier of reset type, with no resistive components in the feedback loop. The feedback capacitor discharging is performed by means of a diode working in pulsed mode. This gives the benefit of lowest possible noise, high pulse rate capability and the ability to measure the Si PIN diode leakage current during the mission. The preamplifier is followed by two semi-gaussian pulse shaping amplifiers, one with a fast and one with a slower shaping time constant. The fast shaper is used for pulse triggering and pile-up detection and the slow shaper for energy determination. The 
fast shaper has a pulse-pair timing resolution of less than $2 \mu \mathrm{s}$, resulting in less than $4 \%$ pile-up at an input rate of 20,000 counts/s (Lehtolainen et al. 2014). Simultaneously it will allow triggering of pulses from the required $1 \mathrm{keV}$ low energy threshold. The slow shaper has a shaping time of around $5 \mu \mathrm{s}$ (pulse duration around $25 \mu \mathrm{s}$ ), resulting in the beginning of life (BoL) energy resolution for the detectors of around $150 \mathrm{eV}$ (full width at half maximum, FWHM) at $5.9 \mathrm{keV}$, when cooled below $-5^{\circ} \mathrm{C}$.

The detectors require a high voltage bias of about $100 \mathrm{~V}$. This is generated locally by means of an ordinary fly-back converter with a rectifier diode at the high voltage side. The converter transformer is driven by a constant pulse train and the output voltage, which is adjustable, is arranged through a primary voltage supplied by a digital to analogue converter. The bias supply is a feedback stabilized system which makes it fairly insensitive to changes in power supply voltages, environment temperature and load changes. The rectified output voltage is monitored through a high-ohm voltage divider and the feedback voltage is fed to an error amplifier.

To achieve a proper performance, the Si PIN detectors needs cooling typically to ca. -10 to $-25^{\circ} \mathrm{C}$. The detector temperature needs to be stabilized to at least $\pm 1{ }^{\circ} \mathrm{C}$ accuracy to avoid unnecessary variation in the resolution the system produces. This feature is achieved by using a linear feedback control of the power to the thermoelectric cooler by using the detector temperature which is sensed using the NTC thermistor. A feedback control loop through an operational amplifier stabilizes the detector temperature. The control loop setpoint, i.e. the detector target temperature, is set by a voltage signal supplied by a digital to analogue converter. The cooler can also operate in reverse mode and act as a heater which allows annealing, and partial recovery from the radiation induced damage in the PIN diode.

All digital functions related to controlling and reading out the detectors, as well as data and command transfer to and from the SIXS on-board computer are handled by the firmware programmed in an FPGA.

\subsubsection{Operational Principles}

The X-ray detector system of SIXS consists of three almost identical detector packages units and the main electronics. Each detector unit consists of a detector package which is directly coupled with preamplifier electronics. The main electronics collects and processes the signals from the detector units and provides the necessary power input. It handles one detector unit at a time by means of multiplexing. The sensors in the detector units are based on high purity silicon PIN diodes, which operate by the principle of direct conversion of the incoming X-ray photons into electron-hole pairs. In the energy range of interest for SIXS, photoelectric absorption is the dominant effect. The generated electron-hole pairs are subsequently drifted across the diode active volume by means of an electric field supplied across it. These drifting charges induce therefore a signal in the form of a short pulse on the diode electrodes, which is further amplified and converted into a digital format in the electronics connected to each sensor. The electronics consists of a charge sensitive amplifier, physically situated on the preamplifier electronics, followed by a shaper amplifier and analog-to-digital converter (ADC) situated in the main electronics. The shaper amplifier is used in order to increase the signal-to-noise ratio, and the ADC is converting the peak amplitude of each pulse to digital signal, which corresponds to the energy in the detected X-ray quanta. Over time, the main electronics collects a number of such X-ray quanta and bins them into energy spectra which can be sent to the instrument on-board computer and further to ground in the science telemetry packets of SIXS. 


\subsubsection{Data Products}

The X-ray detector system delivers two types of data: spectrum data and flux data. The spectrum data consists of AD (analog to digital) converted counts in 265 energy channels in the energy range $0 \mathrm{keV}-20 \mathrm{keV}$. The lowest 257 channels are evenly spaced in energy with about $40 \mathrm{eV} / \mathrm{channel}$, and the highest 8 channels have logarithmic energy spacing. The events whose energy is higher than $20 \mathrm{keV}$ are collected to the highest energy channel. The logarithmic rebinning of the energy channels above $10 \mathrm{keV}$ was made for telemetry saving purposes, and also since there are no scientifically interesting line features. In addition to the spectrum of the A/D converted events, spectrum data record also includes the total number of events during the integration. This includes both the A/D converted events included in the spectrum, and the events that were detected and counted after the fast shaping amplifier, but not $\mathrm{AD}$ converted. The nominal time resolution of the spectrum data is 16 seconds. The flux data contains the total number of counts, including both the AD converted events and the events that were counted after the fast shaper but not AD converted, with 1 second time resolution. The high time resolution of the flux data allows identification of the periods of spectral data where the incoming X-ray flux has changed significantly during the spectrum integration.

\subsection{Particle Detection System}

\subsubsection{Description}

The particle detector is a closely packed configuration of five $150 \mu \mathrm{m}$ thick Si PIN detectors and a $5 \times 5 \times 6.3 \mathrm{~mm}^{3} \mathrm{CsI}(\mathrm{Tl})$ scintillator with photodiode read-out. It detects electrons in the range $50 \mathrm{keV}$ to $3 \mathrm{MeV}$ and protons in the range 1 to $30 \mathrm{MeV}$ with a total nominal geometric factor of about $0.19 \mathrm{~cm}^{2}$ sr. The core scintillator and its surrounding Si detectors work in coincidence by the $\Delta \mathrm{E}$ vs $\mathrm{E}$ principle for particle identification. Each Si detector is surrounded by an anti-coincidence detector which is built on the same Si chip. A crosssection of the particle detector design is shown in Fig. 8. Pictures of various parts of the flight model without the collimator installed are shown in Fig. 9.

The light yield of CsI(Tl) scintillator is temperature dependent. To achieve better stability and performance over the operational temperature range, a thermal control was included in the subsystem. The heater is a simple pulse width modulated transistor switch, driving current through the resistor at the core detector. The pulse modulated control signal is generated by the FPGA (Field Programmable Gate Array) of the main electronics, based on adjustable temperature target value and actual scintillator detector temperature which is sensed using an NTC thermistor.

In addition to the detector head, the particle detector subsystem also contains full spectroscopic chains of amplifiers for each detector element, AD converter, high voltage supply and all necessary control functions.

The pulse shaping electronics and AD converter are implemented in the ASIC (Application Specific Integrated Circuit) specifically developed for the SIXS particle detector system. The ASIC used in the flight model of the instrument is the second version in which circuitry was further developed to achieve the performance requirements. The ASIC includes 6 identical amplifier channels which are used for the Si PIN detectors (5 side detectors and 1 channel for the sum of the anticoincidence detectors) and two alternative amplifier channels for the CsI(Tl) scintillator channel: low gain and high gain channel of which the latter was used. The ASIC has been manufactured in a $0.35 \mu \mathrm{m}$ CMOS (Complementary Metal Oxide 


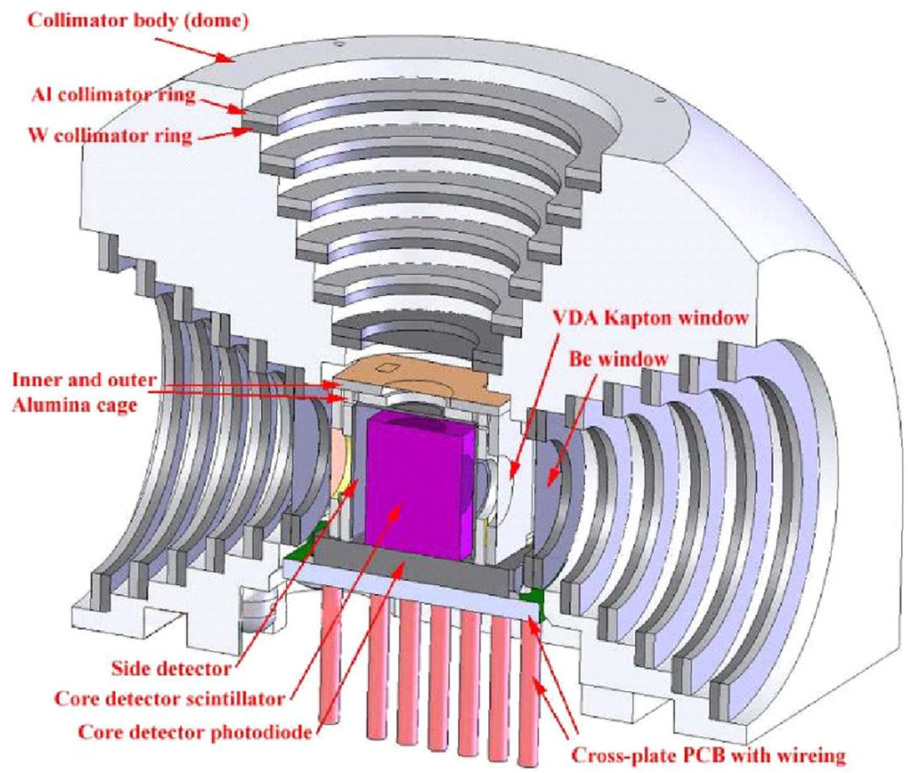

Fig. 8 Cross section of SIXS particle detector system

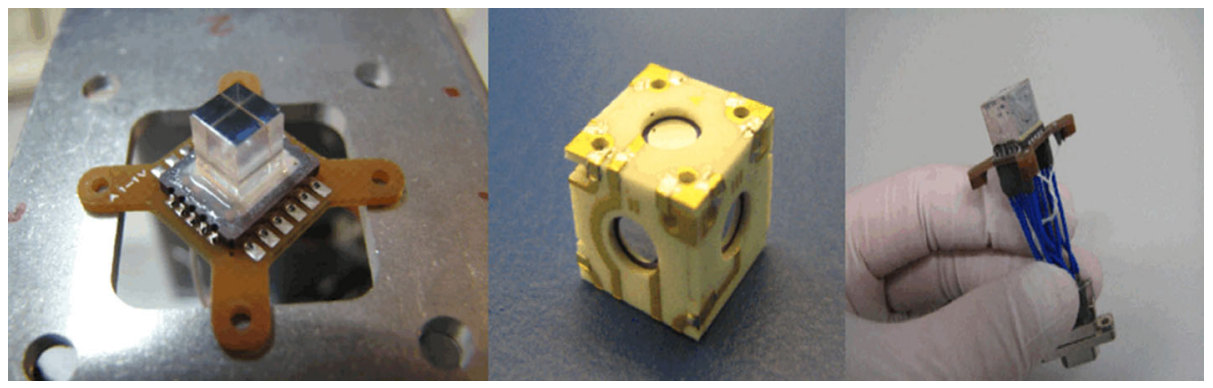

Fig. 9 SIXS particle detector scintillator-photodiode-PCB assembly (left), inner cage including the Si side detectors (middle) and final assembly lacking the collimator structure (right)

Semiconductor) process using enclosed geometry transistors. The resilience against single event effects (SEE) was achieved by using triple voting logic in all digital logic. To improve further the SEE protection, a power regulator providing latch-up protection circuitry is used.

The bias voltage for the detectors is generated by a diode cascade driven by two operational amplifiers. The diode cascade is a common bias source for all the detectors. The source generates a common primary voltage that is distributed to the individual detectors through resistor-capacitance-chains (passive low pass filtering).

The particle detector subsystem is controlled by a digital logic implemented in the FPGA (Actel RT54SX72) operating at frequency of $12 \mathrm{MHz}$. The hit logic control is implemented in the FPGA. The logic is based on the hit signals from each detector indicating that a pulse height has exceeded an adjustable threshold value of a given detector. The FPGA controls the multiplexer and the AD converter in the ASIC to initiate digitization of the detector pulses from the ASIC. Since the conversion of only two detector pulses can be done, and 
provide meaningful particle energy measurements, the selection of the detectors is done in prioritized order which is from the Core detector via the side detectors to the anticoincidence detector. Hence the hit logic always produces pulse heights for combination of any Side and Core detector or any Side detector alone. However, some special hit logic conditions were implemented for calibration and testing purposes.

In addition to the detector pulse digitization, the FPGA controls electronics initiation and parameter setting for the ASIC, House Keeping data collection, data and command interface, and the core detector heater.

\subsubsection{Operational Principles}

The particle detection system of SIXS consists of detector unit and electronics. As described above, the detector unit hosts altogether six detectors: five silicon detectors with two active areas each, and a thallium-doped cesium iodide scintillator with photodiode readout. The photodiode reads the scintillator (called the Core detector) from one side and the other sides are covered by the five silicon detectors. The silicon detectors consist of a central circular active area (with a $2.5 \mathrm{~mm}$ diameter, called Side detectors) and the surrounding active area with rectangular outer form. The surrounding areas of the five silicon detectors are all electrically connected to form one Anticoincidence $(A C)$ detector around the scintillator. The side detectors are covered from above with two thin foils (made of aluminized Be and Kapton) and the view cones are defined with a passive collimator structure, made of aluminum with sandwiched $\mathrm{Al}+\mathrm{W}$ collimator rings defining the view cones and limiting back-scattering of electrons from the collimator walls to the detectors. The foils set the threshold energies of particles able to trigger the Side detectors $(\sim 50 \mathrm{keV}$ for electrons, $\sim 1 \mathrm{MeV}$ for protons). The pulse heights read out from the detectors are proportional to the energy loss suffered by the particle in the detector.

Each incident particle, therefore, can produce signals in up to seven detector channels (five Side detectors, the Core detector, and the AC detector). Particle events contributing to the nominal energy channels of the instrument consist of those, which trigger either one Side detector, only, or one Side detector plus the Core detector. In the former case, the energy measurement performed by the Side detector under the foil system produces a count in the total energy spectrum that can be divided into channels that are primarily counting electrons (below $\sim 300 \mathrm{keV}$ ) and protons (above $\sim 300 \mathrm{keV}$ up to $\sim 4.3 \mathrm{MeV}$ ). In the latter case, two pulse heights are available, which yield both the particle species (electron/proton) and the total energy (above $\sim 300 \mathrm{keV}$ for electrons and $\sim 4.3 \mathrm{MeV}$ for protons) of the incident particles stopping in the core (the $\Delta E$ vs. $E$ method), thus producing a count in the respective electron/proton channels of the instrument. The nominal energy range for electrons (protons) extends up to 3 (30) MeV. Particles at higher energies are collected in integral spectral channels.

\subsubsection{Data Products}

The particle detection system delivers three types of data: spectral data, counting rate data and pulse height data. Spectral data consist of counts in seven (nine) energy channels responding primarily to electrons (protons), taken separately in the five viewing directions of the instrument. The nominal energy channels are spaced quasi-logarithmically in energy, but especially the electron channels are quite broad in their energy response (see Sect. 3.2.2). The time resolution of the spectral data product is nominally 8 seconds. The counting rate data is obtained with 1 second time resolution in broader energy channels (two per species) 
without the angular resolution, i.e., combining counts from all directions into the same omnidirectional channels. The pulse height data contains a sample of both the single (Side) detector particle events and coincidence events (Side+Core), which can be used to monitor the quality of the data and calibration of the instrument, and to reconstruct high-resolution energy spectra for the species at long integration times. The pulse heights represent energy-loss measurements at 11-bit resolution, enough to reconstruct the spectra at the maximal energy resolution of the instrument. The time resolution of the pulse height data is 64 seconds, but since the sample is small, up to six single Side-detector events and ten Side+Core-detector coincidence events, the integration time for a meaningful sample of pulse height data is much higher (up to 21600 samples per day can be collected).

\section{Scientific Performance}

\subsection{X-Ray Detection System}

\subsubsection{Simulations}

SIXS X-ray detectors are based on the heritage of the X-ray solar monitors (XSM) flown on-board ESA's SMART-1 and Chandrayaan-1 missions (Alha et al. 2008, 2009). The most significant differences between SIXS-X detectors and the heritage instruments are in the read-out electronics, and in the sizes and thicknesses of the detector chips and filters. The performance and radiation effect estimate of the SIXS-X detectors were based on the data collected with the heritage instruments and theoretical calculations of the differences between them and the SIXS-X detectors. An exception to this is the design of the in-flight calibration sources and their assemblies, which was based on Monte-Carlo simulations. In the heritage instruments the in-flight calibration sources were fitted on the shutter mechanism in front of the detector's Be entrance window so that they were only visible to the detector when the shutter was closed. This design was not possible for SIXS-X detectors due to a requirement that the instrument shall not contain any moving parts because of the risk of mechanical failure during the long mission. Thus, the calibration sources had to be re-designed and placed inside the detector cups so that they are always visible to the detector, but do not impair their FOVs. The design process is described in detail in Lehtolainen et al. (2011).

The fields of view of each X-ray detectors have also been simulated. They are shown in an spherical coordinate system in a Mercator-type map in Fig. 10.

\subsubsection{Pre-Flight Calibrations and Characterization}

The pre-flight calibrations, characterization and performance verification campaign of the SIXS X-ray detectors was carried out in November 2012 at the facilities of Oxford Instruments Analytical Ltd., and during May and June 2013 in the X-ray laboratory of the University of Helsinki. The calibration procedures are presented in detail in Lehtolainen et al. (2014). SIXS flight model (FM) and flight spare (FS) sensor units were swapped during the BepiColombo MPO assembly, integration and testing (AIT) campaign in 2014, and the model chosen for flight was the original FS sensor unit. The calibration and performance results presented in Lehtolainen et al. (2014) are those of the original FM. The calibrations of the currently flying and operating FS unit were performed identically with those of the original FM, and they are presented here (see Figs. 11-17). They included determination of 


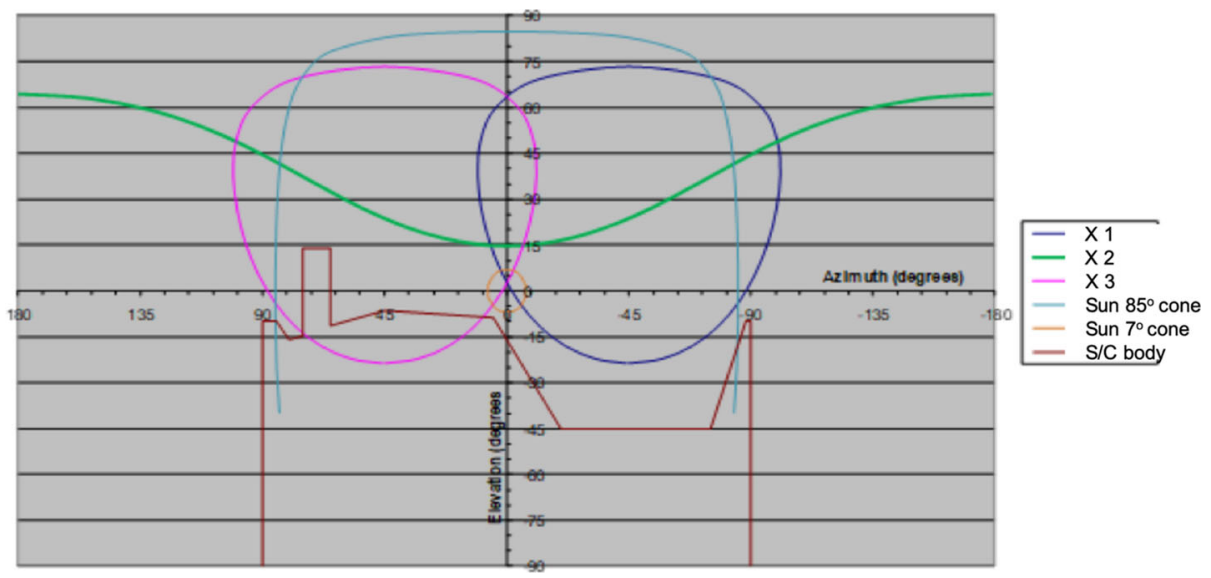

Fig. 10 The Field of View of the X-Ray detector system SIXS-X (3 detectors). The spacecraft (S/C) body is also shown

each detector's sensitivity within its FoV (field of view) as a function of the direction of incident photon and photon energy, determining each detector's BOL (beginning of life) energy resolution as a function of photon energy at different operational temperatures, pile-up tests for determining the pulse pair resolution times of the read-out electronics for each detector, measurements of the low energy thresholds of the energy scales in relation to an adjustable S/W parameter, comparison calibration of the fluorescence line fluxes with the SMART-1 XSM FS detector, and determining the shifting of each detector's energy scale as a function of the temperature of the read-out electronics.

The determination of the sensitivities of each detector included three steps: determination of each detector's sensitive area from the SEM images taken of each detector's aperture hole, theoretical calculation of the transmission of the detectors' beryllium entrance windows, filters and the detectors' sensitive volumes as functions of the photon energy and the angle of incidence (off-axis angle), and the direct measurement of each detector's relative sensitivity as a function of the off-axis angle and the azimuthal roll angle of incoming X-ray radiation by measuring stable X-ray beam from an X-ray tube from different directions. The sensitivity data was measured with 1 degree steps in off-axis direction and 45 degree steps in roll direction. The results, shown in Figs. 11, 12 and 13, were then interpolated and combined to form the FoV sensitivity maps for each detector as a function of the off-axis and roll angles, and photon energy. The sensitivity maps are used in the science data processing pipeline of SIXS-X as a database from which the ancillary response files (ARF) for each Sun observation are selected based on the position of the Sun in the detector's FoV.

Since the SIXS-X detectors contain built-in Ti coated ${ }^{55} \mathrm{Fe}$ in-flight calibration sources for the determination of energy scale and resolution during flight, determination of each detector's BOL energy resolution as a function of photon energy and comparison calibration of the fluorescence line fluxes with SMART-1 XSM FS detector were performed primarily for performance verification purposes. The measurements were made by analyzing the characteristic line spectra from different fluorescence sources with each detector at different detector temperatures. The results are shown in Fig. 14.

The results show that the energy resolutions of SIXS-X detectors are much better than the BOL performance requirement of $250 \mathrm{eV}$ at $6 \mathrm{keV}$, and that at BOL the degradation of energy resolution due to increased leakage current at higher detector temperatures is small. 

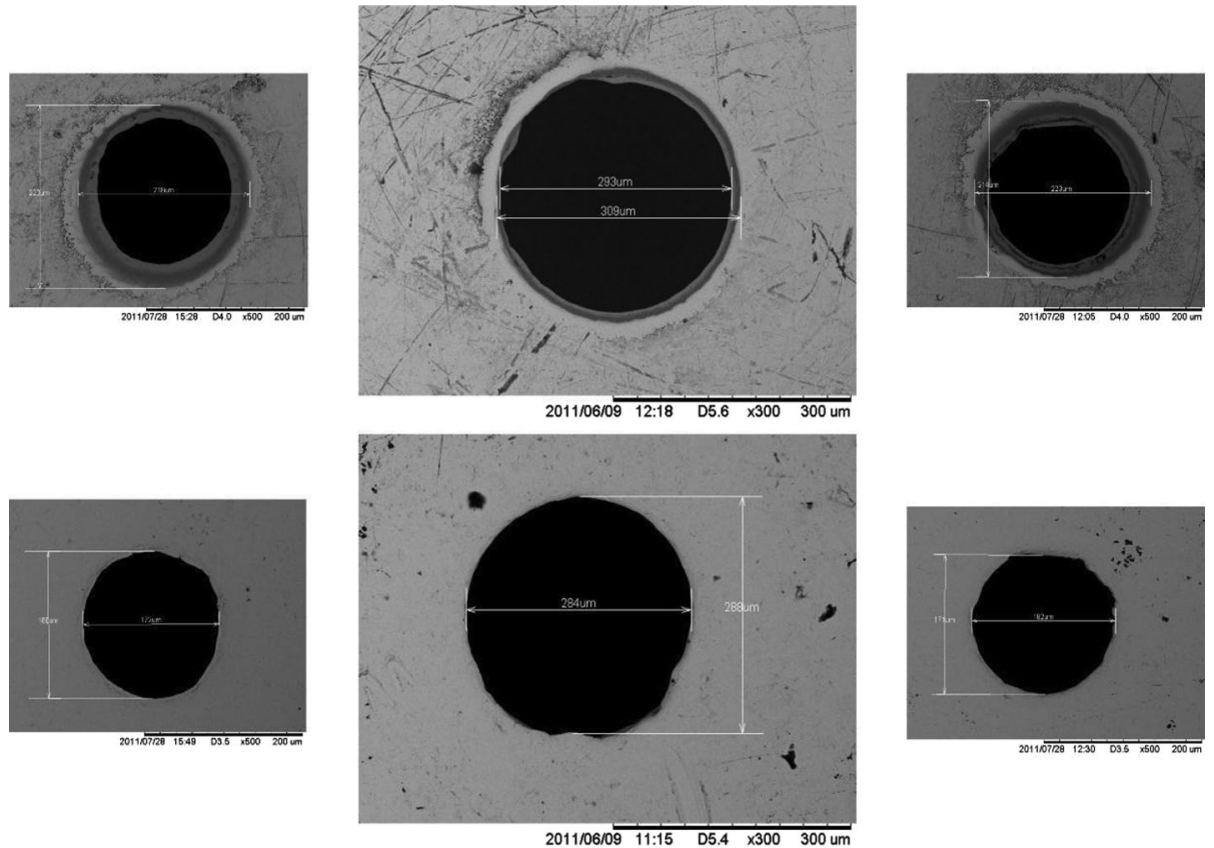

Fig. 11 SEM (Scanning Electron Microscope) images of the aperture holes of the SIXS-X detectors 1 (left), 2 (center) and 3 (right) taken from above/outside (upper figures) and below/inside (lower figures)

This result, combined with the radiation effects analysis made by the detector manufacturer, imply that the energy resolution is expected to remain well within requirements throughout the mission.

The pile-up tests were performed for determining the pulse pair resolution times of the read-out electronics for each detector and ensure that the detectors fulfil the performance requirements for their dynamic range. Theoretical calculations show that an X1 class flare would produce an X-ray signal of about $20000 \mathrm{cps}$ to the larger SIXS-X detector at the perihelion distance of Mercury. Thus, the scientific requirement is that the SIXS-X detectors shall be able to measure an X-ray signal of 20000 cps with less than $4 \%$ pile-up. Poisson statistical calculations shown e.g. in Lehtolainen et al. (2014) show that the requirement is fulfilled if the pulse pair resolution time constant is $<2 \mu \mathrm{s}$. The pulse pair resolution was determined by determining the pile-up probability at different count rates for each detector and fitting the Poisson statistical function for pile-up probability as a function of the count rate to the results to obtain the pulse pair resolution time constants for each detector. The results are shown in Fig. 15.

The pulse pair resolution time constants of all three detectors are $<2 \mu \mathrm{s}$. Thus, SIXS-X detectors fulfil the scientific requirements for the upper limit of their dynamic range.

The low energy thresholds of the energy scales of SIXS-X detectors in relation to an adjustable S/W parameter were found to be identical with those of the spare unit, shown in Lehtolainen et al. (2014). However, the low energy noise filtered out by the low energy threshold, becomes visible in detector 2 at slightly higher threshold setting than in the other detectors. The noise floor in detector 2 was found to be at channel $18(\sim 0.72 \mathrm{keV})$, while in the other two detectors and all three detectors of the spare sensor unit it is at channel 15 $(\sim 0.6 \mathrm{keV})$. The corresponding energy shown in parenthesis depends slightly on tempera- 


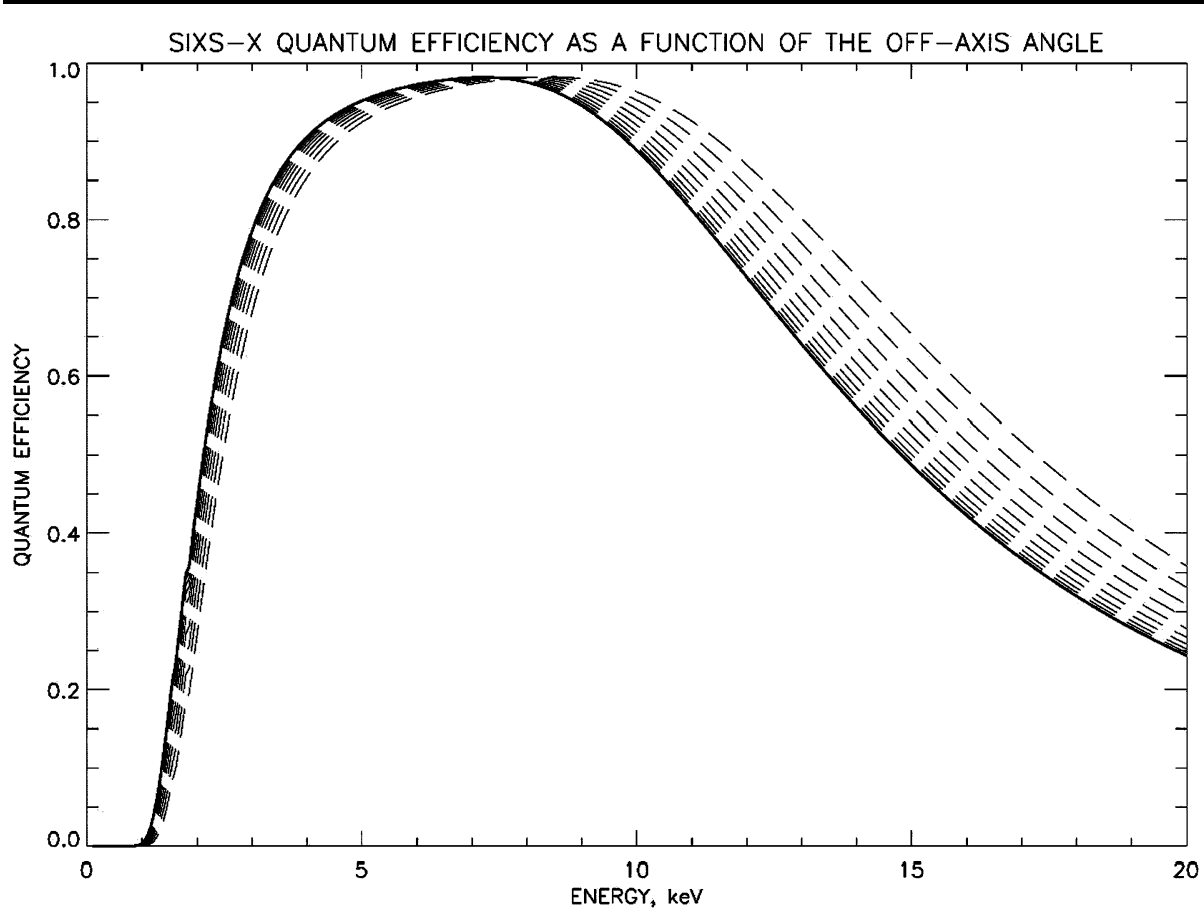

Fig. 12 Quantum efficiencies of the SIXS-X detectors as a function of photon energy plotted with off-axis angles between $0^{\circ}-55^{\circ}$ with $5^{\circ}$ steps

SIXS-X FOV SENSITIVITY MAPS

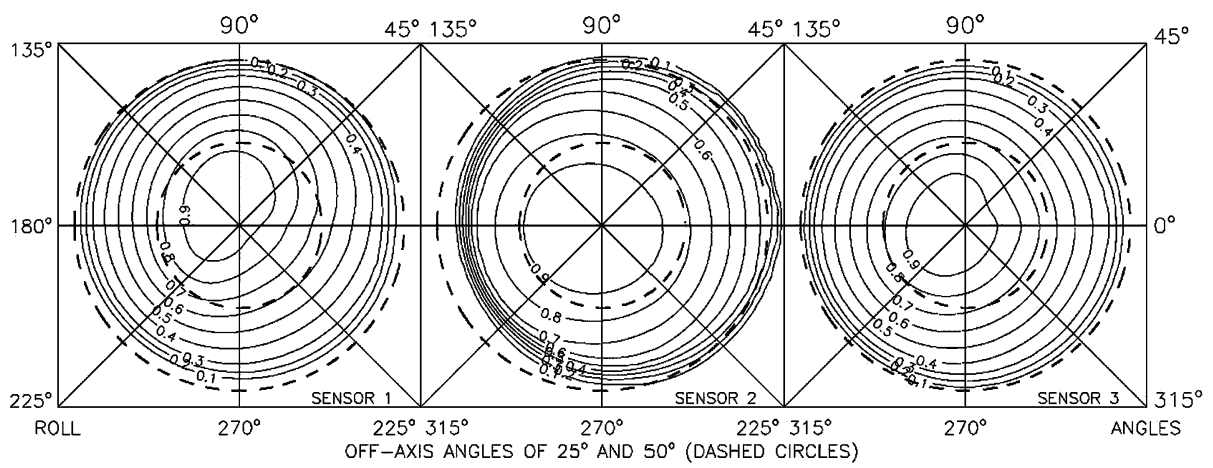

Fig. 13 Relative FoV sensitivity maps of the SIXS-X detectors normalized to maximum (on-axis) FoV sensitivity

ture of the read-out electronics ( $\pm 40 \mathrm{eV}$ within the operational temperature range of SIXS). These values are, however, well below $1 \mathrm{keV}$, which is the practical lower limit of the energy range of SIXS-X as seen in Fig. 12.

Shifting of each detector's energy scale as a function of the temperature of the read-out electronics was investigated by analyzing the signals from the detectors' in-flight calibration sources at different temperatures. A climate chamber was used to stabilize the temperatures. 


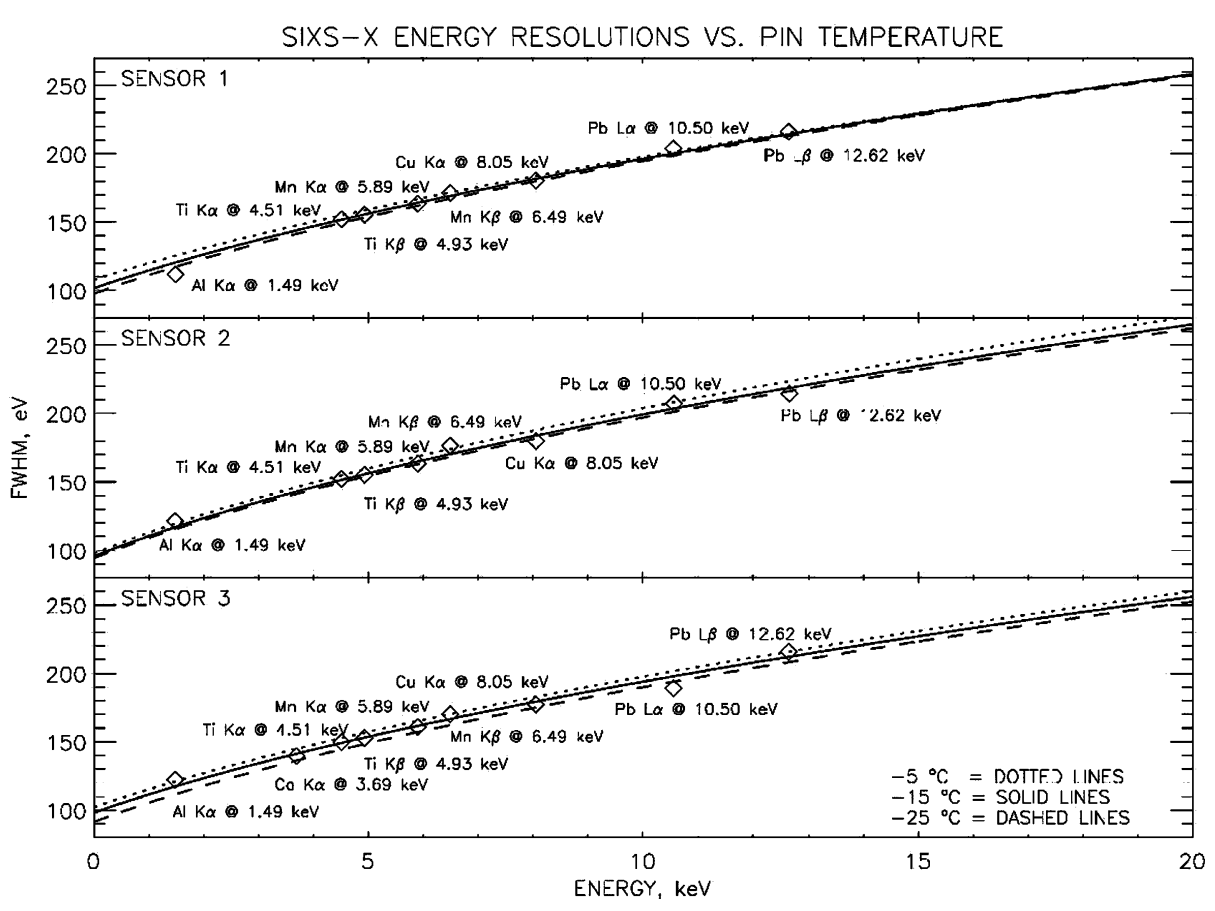

Fig. 14 Beginning-Of-Life (BOL) energy resolutions of the SIXS X-ray detectors (SENSOR 1,2,3) as a function of photon energy at different detector temperatures

The shifting of the energy scale is shown in Figs. 16 and 17, which show each detector's gain and offset as a function of the electronics temperature, respectively.

During the mission the detector gain and offset, as well as the energy resolution, are determined for each observation from the spectral lines of the in-flight calibration sources, which are always visible to the detectors. The data shown in Figs. 16 and 17 will be used mainly to assess the stability of detector gains and offsets during the mission. This information will be important for future space missions with similar X-ray detectors because stable temperature dependencies of gain and offset would enable determination of the energy scale from the temperature, and energy resolution from the leakage current without the use of in-flight calibration sources.

\subsection{Particle Detection System}

\subsubsection{Simulations}

The SIXS particle detection system has been extensively simulated using the Geant 4 Monte Carlo simulation framework to get an accurate understanding of the response of the instrument to particles in the nominal energy range and outside of it (Huovelin et al. 2010; Heino 2016). The simulation model for particles incident outside the apertures but energetic enough to penetrate the collimator structures differs from the model in the nominal range. In the nominal energy range, we simulated particles isotropically inside the cone of half-width of $60^{\circ}$ incident on rectangular plates just outside each of the five the collimator openings. The total geometric factor of the particle source plates can be calculated analytically and the 


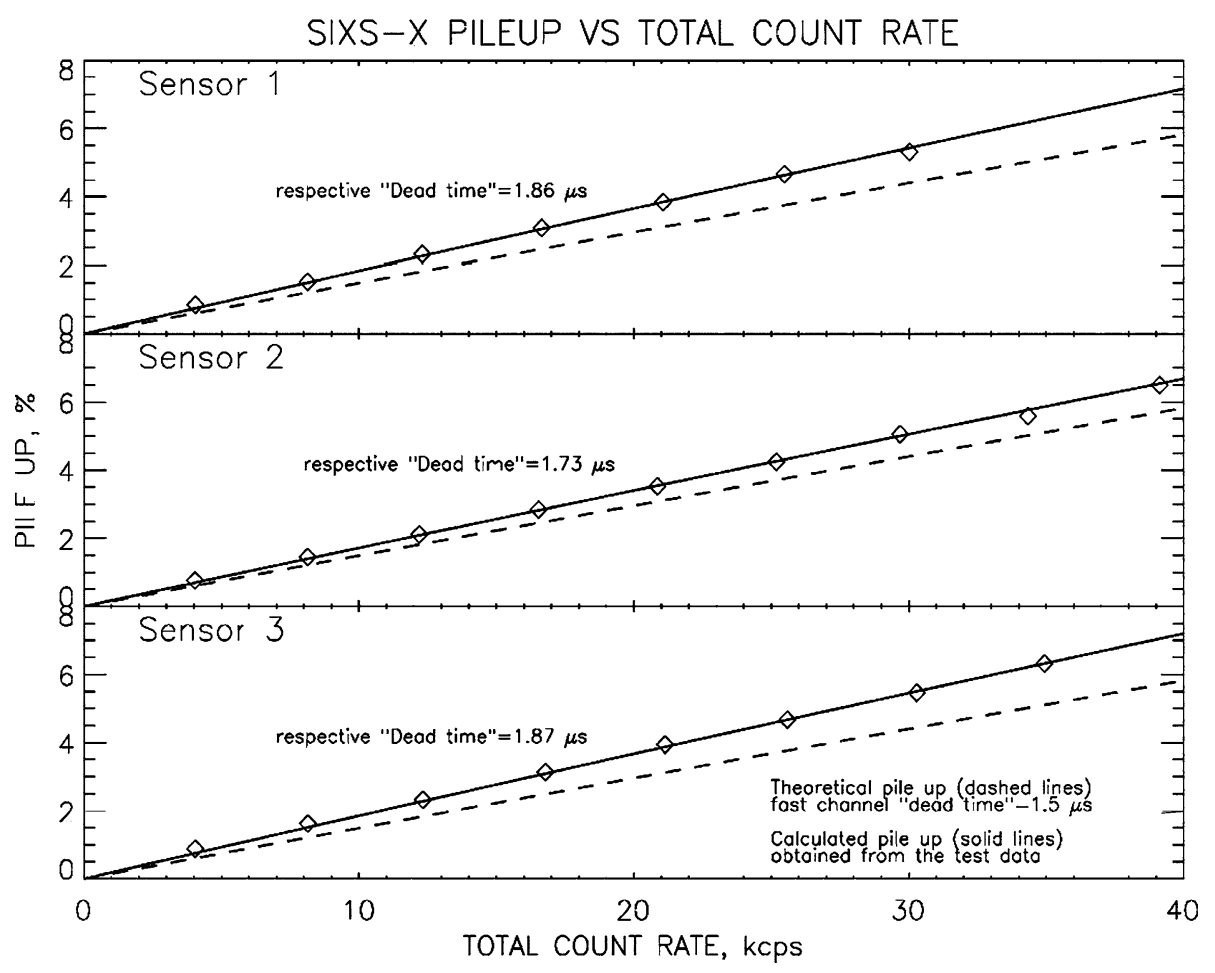

Fig. 15 Measured and theoretical (pulse pair resolution time $=1.5 \mu$ s) pile-up percentages of the SIXS-X detectors as functions of the count rate

geometric factor of the instrument as a function of incident energy can then be obtained as a fraction of particles that would be detected from the total number of simulated particles, multiplied by the geometric factor of the source. For particles penetrating the collimator we opted for using a sector of a spherical particle source surface and an isotropic angular distribution of particles inside a cone around the local zenith that encloses the active detector elements in their entirety.

The proton simulations show that the energy channels have quite close to nominal, box-car-like response as a function of energy and the characteristic channel energies and corresponding geometric factors multiplied by effective channel widths have been listed in Table 2. They are obtained using the Geant4 simulated response functions of each channel and applying the Van Allen bowtie method (e.g., Sorensen et al. 2005; Oleynik et al. 2019) to find the pairs of characteristic energies and geometric factors that best reproduce the intensities from counting rates for a broad range of power-law spectra. The last channel P9 is not really a differential channel so the value of characteristic energy is much less certain than the values obtained for the other channels. The energy range of P9 spans energies from below $30 \mathrm{MeV}$ to the $\mathrm{GeV}$ range, but beyond $\sim 150 \mathrm{MeV}$ protons start to be counted in electron channels, as well.

Electron simulations, however, show that scattering inside the detector produces a relatively broad response of the energy channels. The final values of the energy limits still require the inflight calibration to be performed once an event with enough statistics can be measured, but the simulated responses with information on the detector calibration from ground show that all channels have long tails in their energy response beyond the nominal 


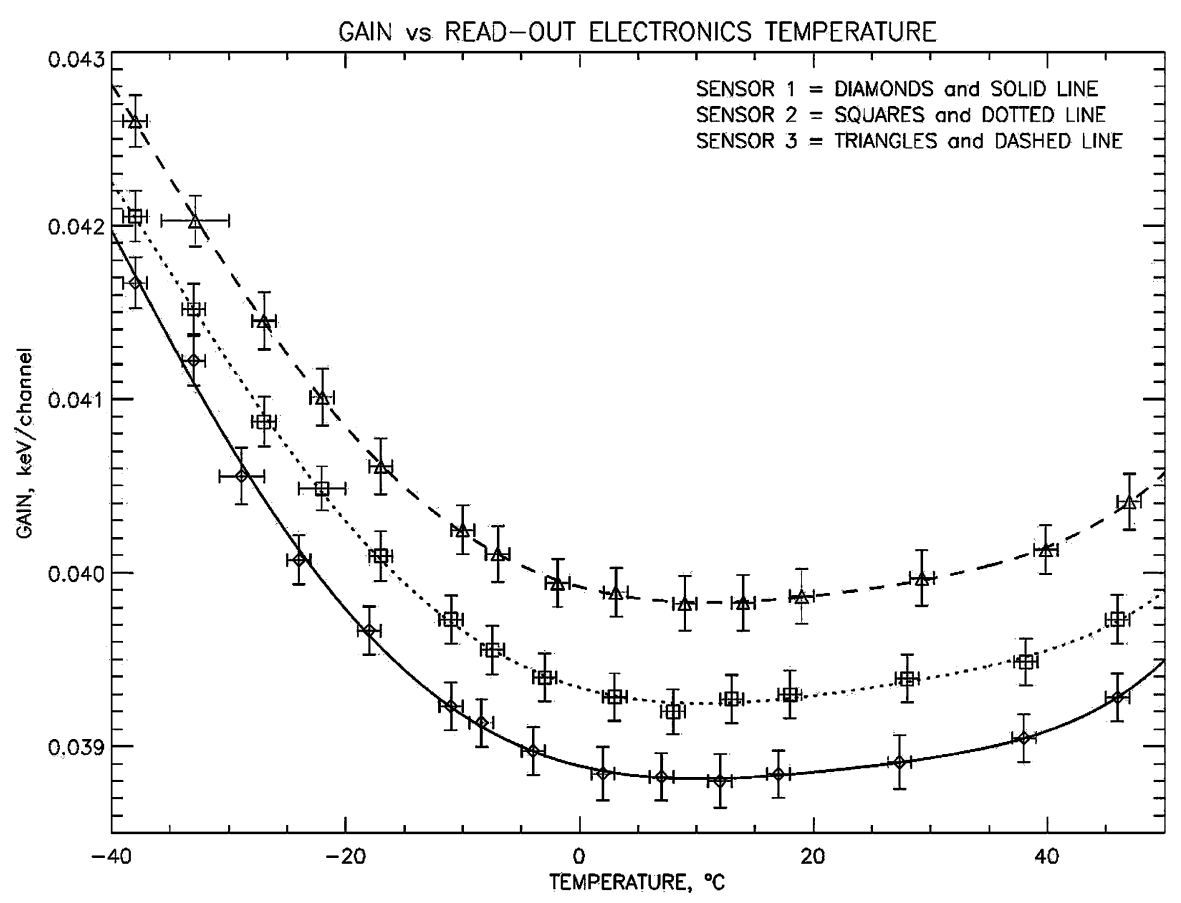

Fig. 16 Energy scale gain shifts of the SIXS-X detectors as functions of the temperature of the read-out electronics

Table 2 Characteristic energies and geometric factors of proton energy channels of SIXS particle detection system. The last channel P9 is very broad so the characteristic energy has a lot of uncertainty that depends on the spectrum, as reflected by the error limits of the $\mathrm{E}_{\mathrm{c}}$

\begin{tabular}{llllllllll}
\hline Channel & P1 & P2 & P3 & P4 & P5 & P6 & P7 & P8 & P9 \\
\hline $\mathrm{E}_{\mathrm{c}}[\mathrm{MeV}]$ & 1.09 & 1.19 & 1.51 & 2.26 & 3.94 & 8.02 & 14.1 & 25.6 & 37 \\
$\mathrm{E}_{\mathrm{c}}-\delta \mathrm{E}_{-}[\mathrm{MeV}]$ & 0.95 & 1.09 & 1.41 & 2.14 & 3.66 & 7.35 & 12.9 & 21.0 & 12.4 \\
$\mathrm{E}_{\mathrm{c}}+\delta \mathrm{E}_{+}[\mathrm{MeV}]$ & 1.25 & 1.31 & 1.61 & 2.38 & 4.22 & 8.32 & 15.6 & 31.2 & 110 \\
$\mathrm{G} \Delta \mathrm{E}\left[\mathrm{cm}^{2} \mathrm{sr} \mathrm{MeV}\right]$ & 0.0033 & 0.0055 & 0.0214 & 0.0371 & 0.105 & 0.177 & 0.275 & 0.779 & 1.69 \\
\hline
\end{tabular}

energy range of the channel. Figure 18 shows the electron channel responses in a simulation with an estimate of the core detection efficiency that electrons are recorded in the core at energy deposits exceeding $500 \mathrm{keV}$, which according to the ground calibration data seems reasonable. The response is for a single unobstructed field-of-view (Side 0 , but the response is very similar in other sides as well). The effective detection threshold has to be determined from inflight data, once a sufficiently strong solar electron event or the Earth's outer radiation belt during the flyby in 2020 can be observed. The electron channels E2 and E5, in particular, are affected by the effective core detection threshold energy. Channel E2 has a broad response without a properly defined mean energy, unless the electron energy spectrum is very steep so that the first peak in the response dominates. The most stable differential measurement will be provided by the E1 and E4 channels nominally at 50-60 keV and 180-300 keV, respectively. Also E5 is a differential channel, but its energy range depends 


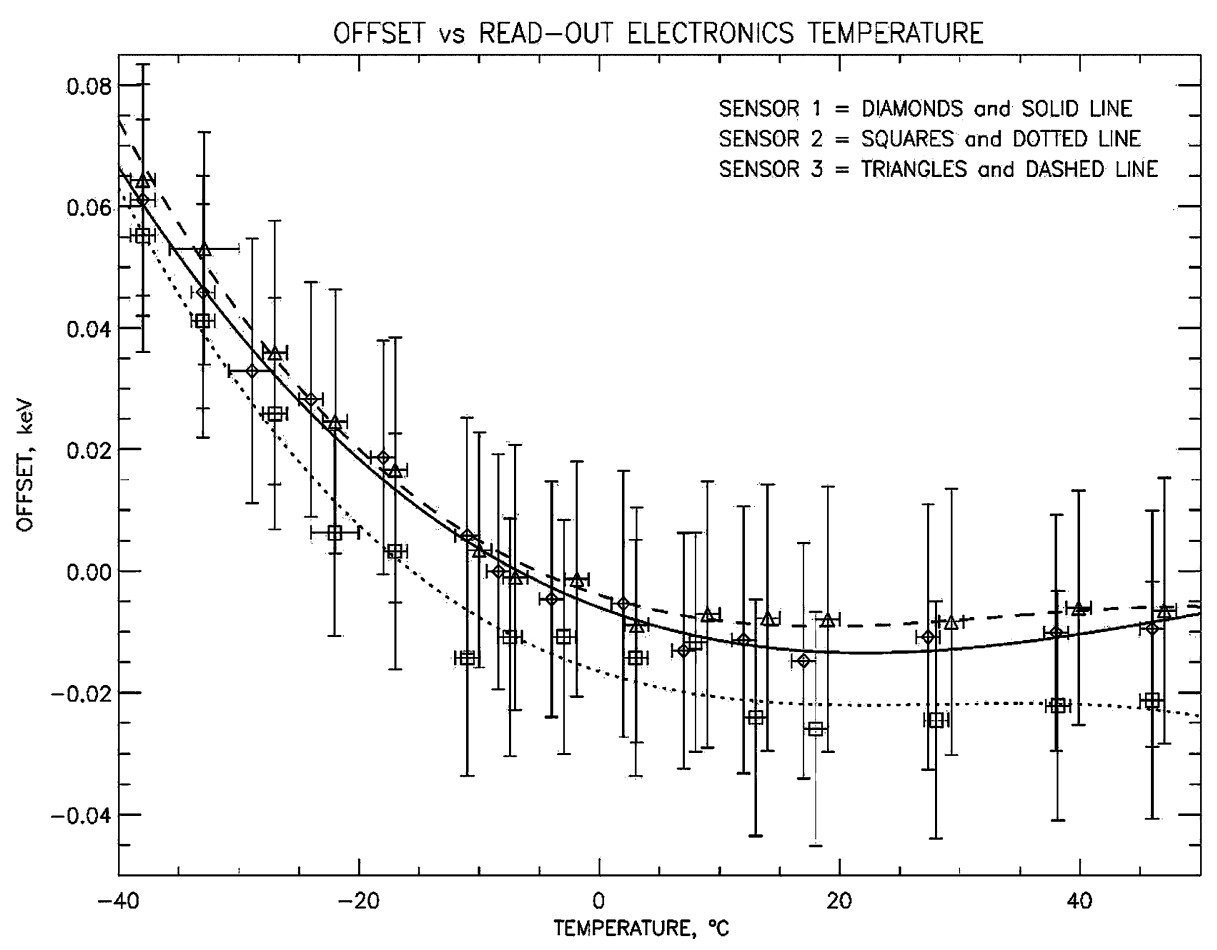

Fig. 17 Energy scale offset shifts of the SIXS-X detectors as a function of the temperature of the read-out electronics

strongly on the core detection efficiency and can be given only after the inflight calibration with electrons. E6 and E7 will provide well-defined integral channels above 1.3 and $3 \mathrm{MeV}$, respectively.

To get to the counting rates, $C_{j}$, the response functions $R_{j}(E)$, presented in Fig. 18 for the electron channels $j=1,2, \ldots, 6$ need to be multiplied with the electron energy spectrum $d J / d E$ and integrated over energy. Figure 19 presents the channel responses per logarithmic energy interval for an example spectral shape fitted by Heino (2016) to a large electron event observed by MESSENGER/EPS on 4 June 2011. The counting rates in the channels can be obtained as proportional to the area under each curve in this plot. It can be seen that SIXS is able to cover the whole energy range from $50 \mathrm{keV}$ to $3 \mathrm{MeV}$ with channels showing comparable counting statistics. This indicates that the detection system will be suitable for the determination of particle spectra in the targeted energy range. The plot also confirms that for a typical spectrum, at least, some of the electron channels will not have a simple response with a well-defined mean or median energy. It suggests, however, that the effective electron detection threshold of the core detector can be obtained once a strong enough electron event, showing increase in all channels, is observed. Channels E1, E4 and E6 offer a quasi-logarithmically spaced set of energy channels that can deliver the initial estimate of the spectral form, which can then be used for the iteration of the core detection threshold affecting mostly channels E2, E3 and E5. After the threshold has been determined, we can evaluate again, which of the spectral channels are most differential in their response and use those as a starting point to deliver the electron spectra. 


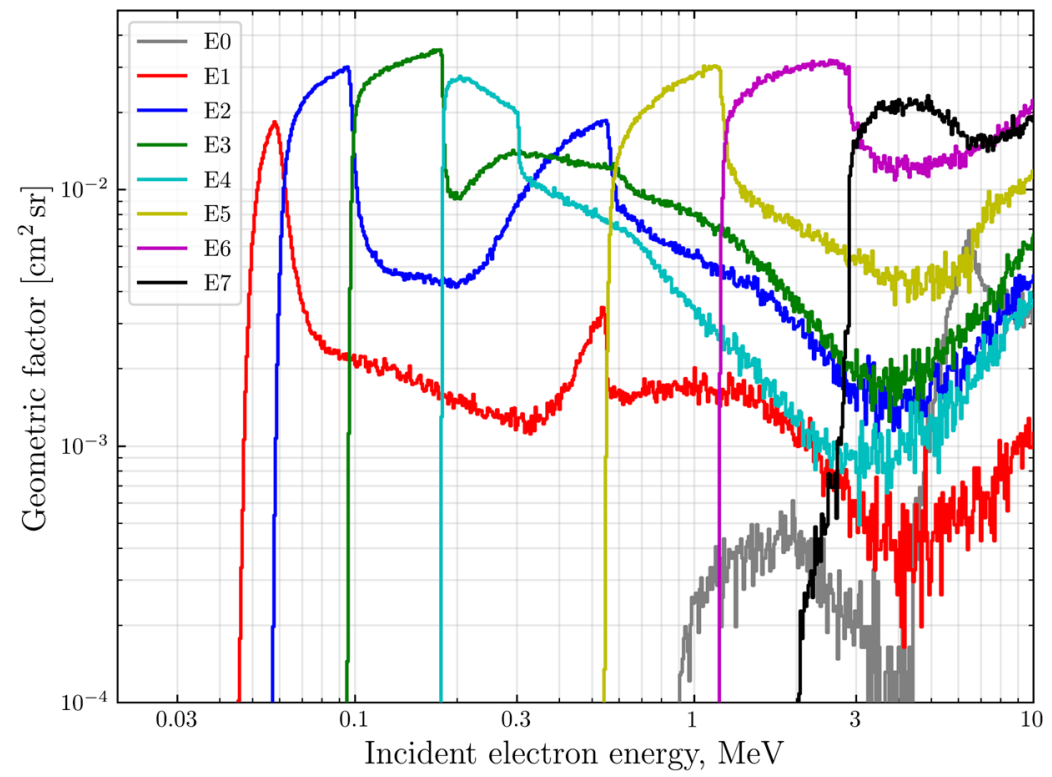

Fig. 18 Geant4-simulated response functions of electron channels E1 to E7 assuming the energy responses of the detector elements based on ground calibrations and an estimate of the scintillator detection threshold energy of $500 \mathrm{keV}$. The response is given for one unobstructed field of view (Side 0). E0 denotes electrons not counted in any of the channels

Since the lowest energy channels for both protons and electrons rely on single detector measurement, there is unavoidably also some cross-talk between the channels. Protons at $\sim 0.9-1 \mathrm{MeV}$ can contaminate the electron channels E2-E4 and electrons at $>200 \mathrm{keV}$ can contaminate proton channels P1 (strongly) and P2 (somewhat). Electron channels E1 and E5 are practically clean from proton contamination and proton channels P3-P8 are clean from electron contamination. P9 can be contaminated with $>3 \mathrm{MeV}$ electrons and E6 and E7 by very high-energy protons (>150 MeV).

Also the fields of view of the particle detectors have been simulated. They are illustrated in spherical coordinates in a Mercator map shown in Fig. 20.

\subsubsection{Pre-Flight Calibrations and Characterizations}

The particle detection system was calibrated for protons using particle accelerator beams in the University of Helsinki (Kumpula) and University of Jyväskylä (RADEF). The beam energies were varied between 1.5 and $55 \mathrm{MeV}$, covering the nominal energy range of the instrument and the first part of its extension to the integral energy range. Electrons were calibrated at the lowest energies $(45-80 \mathrm{keV})$ in the calibration facility of the University of Kiel and at higher energies (384-1234 keV) using radioactive sources and a magnetic spectrometer to select the rigidities. The calibration runs allowed for the accurate determination of the energy response of the detector elements and confirmation of the simulated energy-loss patterns of particles in the detector. In addition, they confirmed that the foreseen maximum counting rates of $20 \mathrm{kHz}$ can be handled by the electronics without significant degradation of the performance (unrecoverable saturation or pulse pile-up effects). From the ground calibrations, however, it was not possible to accurately determine the scintillator detection 


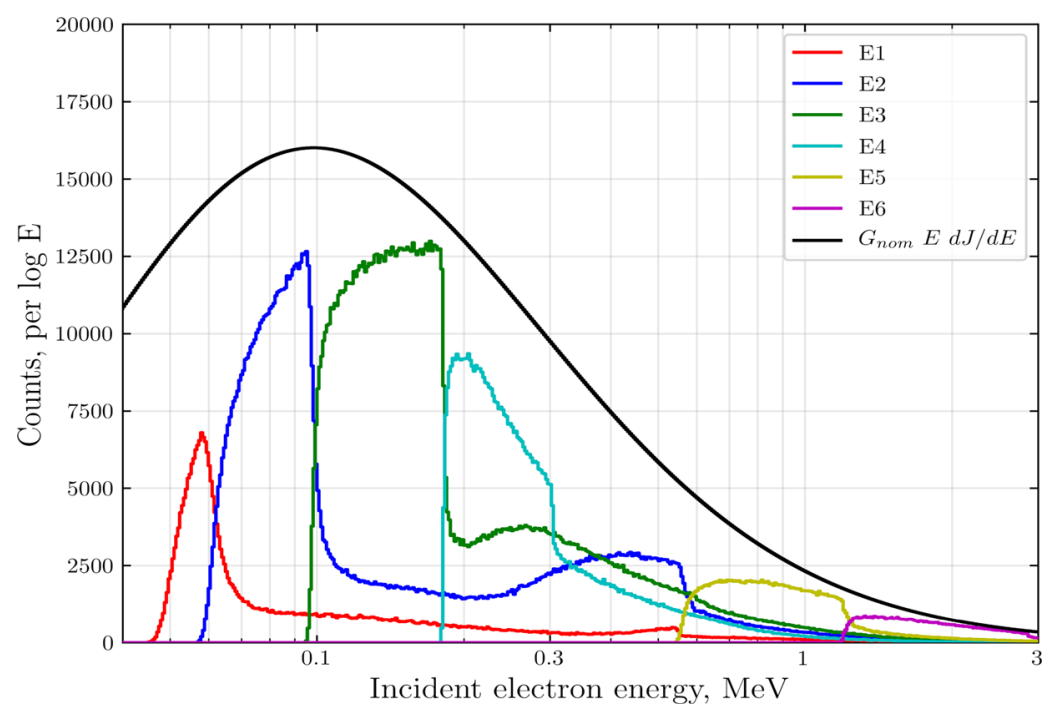

Fig. 19 Differential response of the SIXS particle detection system to an electron spectral shape observed by MESSENGER/EPS during a solar electron event on 2011-06-04. Each curve represents the response function of Fig. 18 multiplied by $E \mathrm{~d} J / \mathrm{d} E$ and the black curve is the same multiplied by the nominal geometric factor of the view cone, $G_{\mathrm{nom}}=0.038 \mathrm{~cm}^{2} \mathrm{sr}$. The visual area under each curve is proportional to the counts observed in the channel. (Note the logarithmic energy axis)

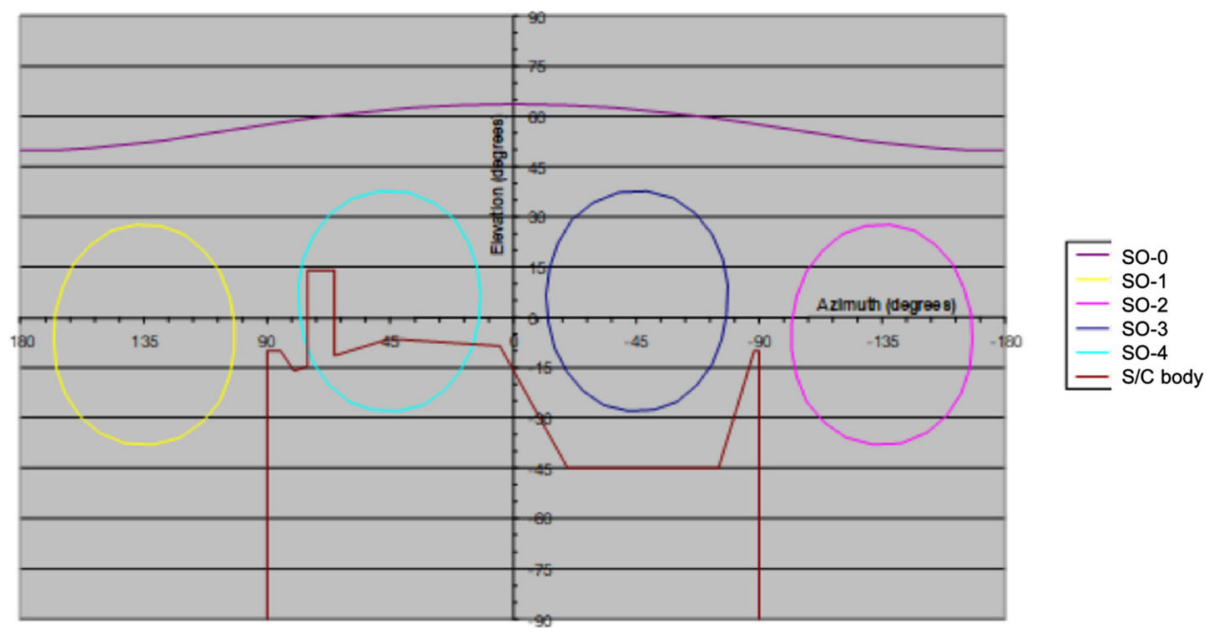

Fig. 20 Field of View (FoV) of SIXS-P particle detector system (5 detectors, SO-N, N =0, 1, 2, 3, 4). The spacecraft $(\mathrm{S} / \mathrm{C})$ body is also shown

efficiency at the lowest pulses caused by electrons. In the radiation source measurements with magnetic filtering, pulses in the scintillator from $456 \mathrm{keV}$ electrons (incident energy) were detected, but the majority of the electrons at this energy triggered the side detector, only. A clear peak in the scintillator was detected at $729 \mathrm{keV}$, but even at that nominal electron energy a significant fraction of electrons do not trigger the core. Fully resolved peak in 


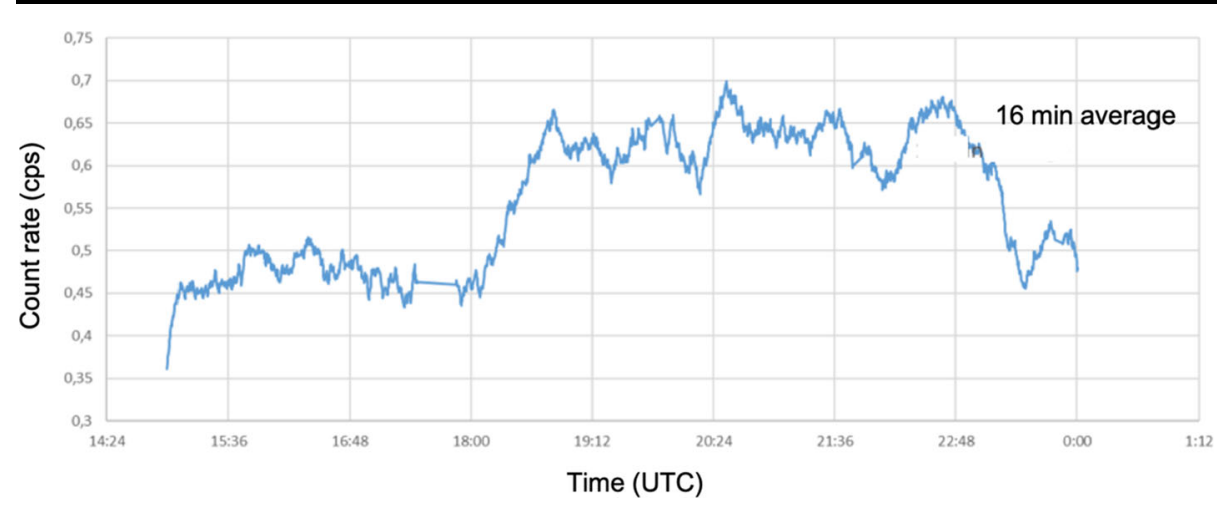

Fig. 21 SIXS-X detector 1, 16 min sliding average raw count rate including channels between 1-2 keV on June 4th 2019. Spacecraft slew maneuver was initiated at 17:57 and completed at 18:47 (UTC). Slew reversion began at 22:57 and ended at 23:47. Increased count rate during the maneuver beginning from about 18:00 is due to the emergence of the Sun in the detector's FoV. The background count rate of $0.45-0.5 \mathrm{cps}$ is primarily due to the low energy tail from the in-flight calibration source in the spectral window

the core detector (most likely corresponding to a $100 \%$ detection efficiency) was obtained at $957 \mathrm{keV}$ electron energy. However, the magnetic spectrometer we used passes, in addition to the selected energy, quite a lot of scattered lower-energy electrons, so it was not possible to accurately estimate the effective detection threshold from the ground calibration measurements. This will be revisited once the instrument has detected a large solar electron event or after the Earth fly-by taking it through the radiation belts.

\section{In-Flight Performance and First Results}

\subsection{X-Ray Detection System}

In a nominal cruise phase attitude the SIXS-X detectors' view to Sun is blocked by the sun cover (MOSIF) of the Jaxa's MMO (Murakami et al. 2020). In order to bring the Sun into the FoV of SIXS-X, the entire spacecraft stack must be rotated by $15^{\circ}$ around the spacecraft $\mathrm{Z}$-axis. Thus, during most of the cruise phase, SIXS-X can measure only the X-ray sky excluding the Sun. However, the needed slew maneuver was performed on June 4th 2019. The maneuver was initiated at 17:57 (UTC) and completed at 18:47. The slew reversion began at 22:57 and ended at 23:47. SIXS-X was observing with detector 1 throughout the maneuver.

The distance from BepiColombo to the Sun was 1.17 AU and the solar X-ray flux measured by GOES was flatlined at quiet A8 level $\left(8 \cdot 10^{-8} \mathrm{~W} / \mathrm{m}^{2}\right)$ throughout the observation. Therefore, the X-ray flux was over an order of magnitude below the effective lower limit of the dynamic range of SIXS-X since its designed lower limit is at B1 level near Mercury ( $~ 0.4 \mathrm{AU})$, and also the detector that had the Sun in its FoV is one of the two detectors with the smaller effective areas. Thus, the observed solar signal was very weak. The 16 minutes long sliding average raw count rate within 1-2 keV spectral window is shown in Fig. 21.

Due to the weakness of the solar signal it was necessary to bin the individual spectra from a longer time window together to resolve the time variability and the solar X-ray spectrum with sufficient statistics. The entire $4 \mathrm{~h}$ dataset from 18:47-22:57 (UTC) was summed up after compensating for the temperature induced shifting in the detector gain and offset, and 


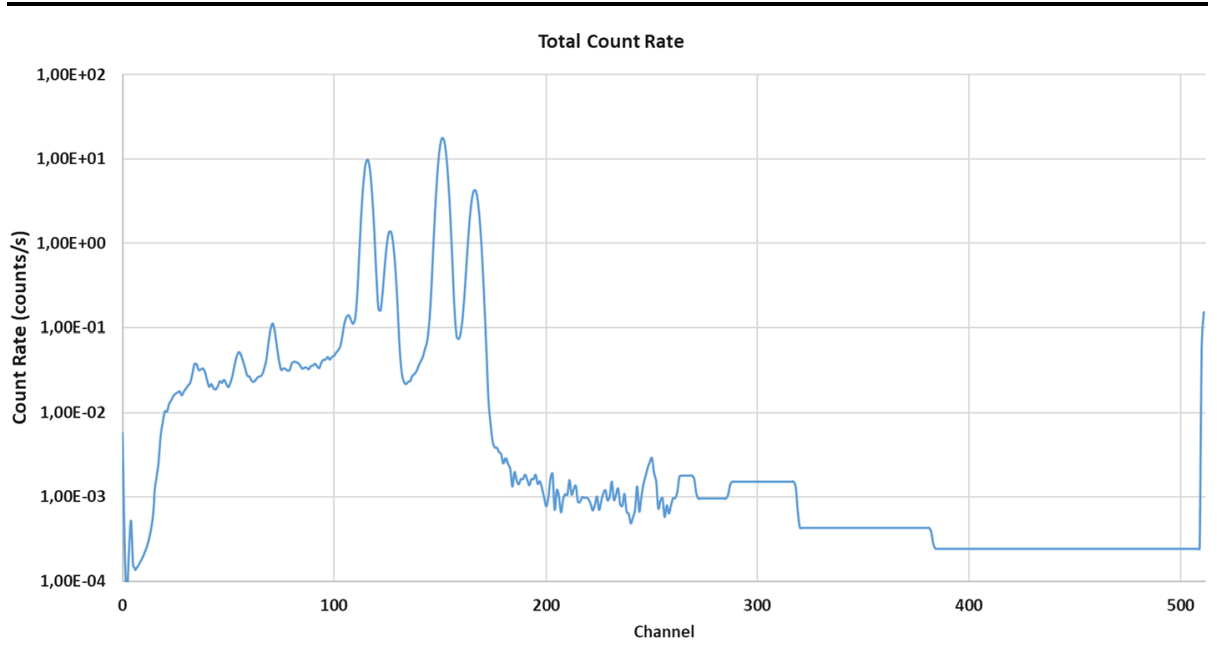

Fig. 22 Sum spectrum of the Sun observation on June 4th 2019 from 18:47 to 22:57 UTC

further processed as a single spectrum. The temperature dependencies of the detector gain and offset were accounted for by determining the gain and offset for each individual $16 \mathrm{~s}$ spectrum from the spectral lines of the in-flight calibration source and rebinning the individual spectra to a fixed energy scale before summing them up. Both gain and offset were quite noisy, and 2nd order polynomial fits were made on them for during the observation period. The rebinned sum spectrum is shown in Fig. 22.

The dominating contribution of the in-flight calibration source was removed by rebinning a $4 \mathrm{~h}$ observation of empty sky background with the same detector performed earlier on June 4th 2019 to the same energy scale and subtracting the empty sky from the Sun spectrum. This is shown in Figs. 23 and 24. The same empty sky spectrum was also used for refining the detector background model for Detector 1, which is an important part of the SIXS-X science data processing pipeline. Similar background measurements are planned for Detectors 2 and 3 in November and December 2019.

The raw spectrum of solar X-rays was then processed with the SIXS-X science processing pipeline. The ARF was selected from the ground calibration results based on the position of the Sun in the detector's FOV, which remained fixed throughout the observation, and the RMF (Response Matrix File) was determined from the spectral lines of the in-flight calibration source in the sum spectrum. The Gaussian redistribution of photons due to the finite energy resolution and escape of characteristic X-rays from the detector's active area were accounted for in the RMF.

The solar X-ray spectrum shown in Fig. 24 was analyzed by fitting the VAPEC (Variable Astrophysical Plasma Emission Code) model to the data. This is a model for calculating the emission from an optically-thin thermal plasma that allows modelling with variable elemental abundances. The resulting solar spectrum in physical units is shown in Fig. 25 and the model parameters are shown in Table 3. A Description of the VAPEC model can be found at https://heasarc.gsfc.nasa.gov/xanadu/xspec/manual/node134.html.

The temperature and emission measure are at normal levels during quiescence. The observed elevated abundances of $\mathrm{Mg}$ and $\mathrm{Si}$ can be attributed to the FIP effect, which causes elements with low first ionization potential (FIP) to be enriched in the solar corona. This effect is well known (see e.g., Feldman and Widing 2003), but still not well understood. The measured X-ray flux within the nominal GOES low energy channel 1.55-12.4 keV, scaled 


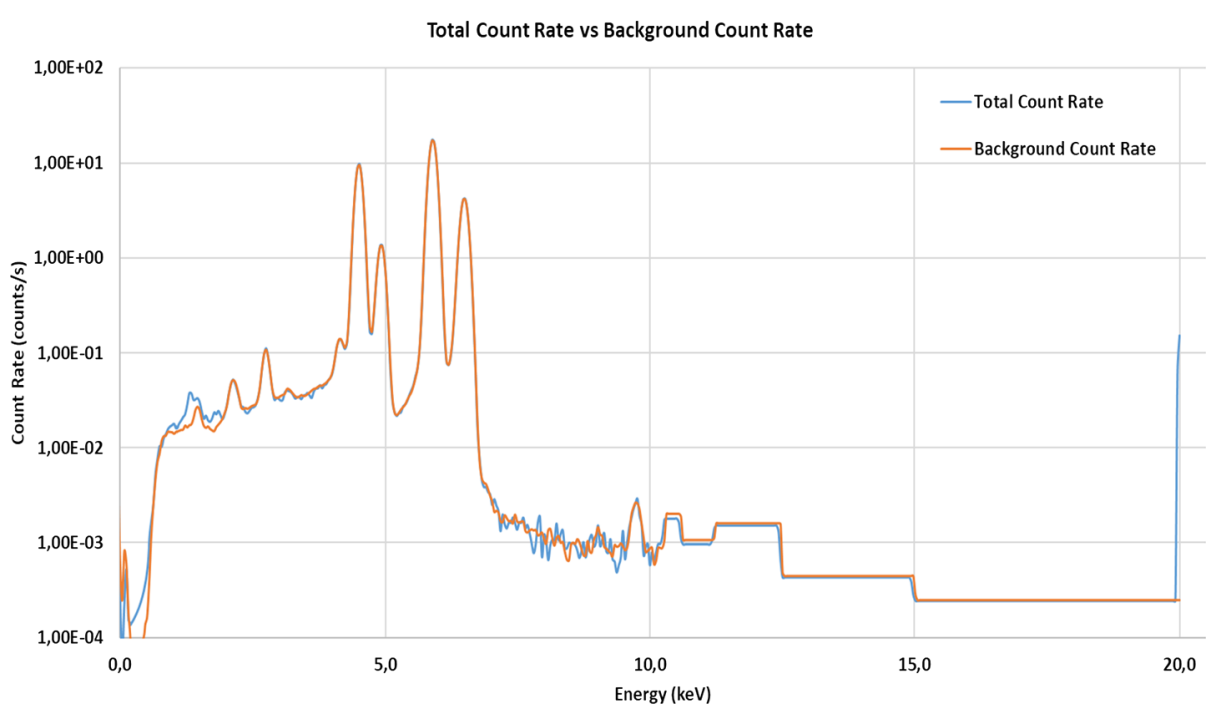

Fig. 23 Comparison of the sum spectrum during the Sun observation and the empty sky spectrum. The peak at $20 \mathrm{keV}$ in the Sun spectrum is caused by solar electrons that deposit more energy to the detector than the upper limit of its energy range. The signal from the Sun is detectable only at energies below $2 \mathrm{keV}$, which is typical during quiescence

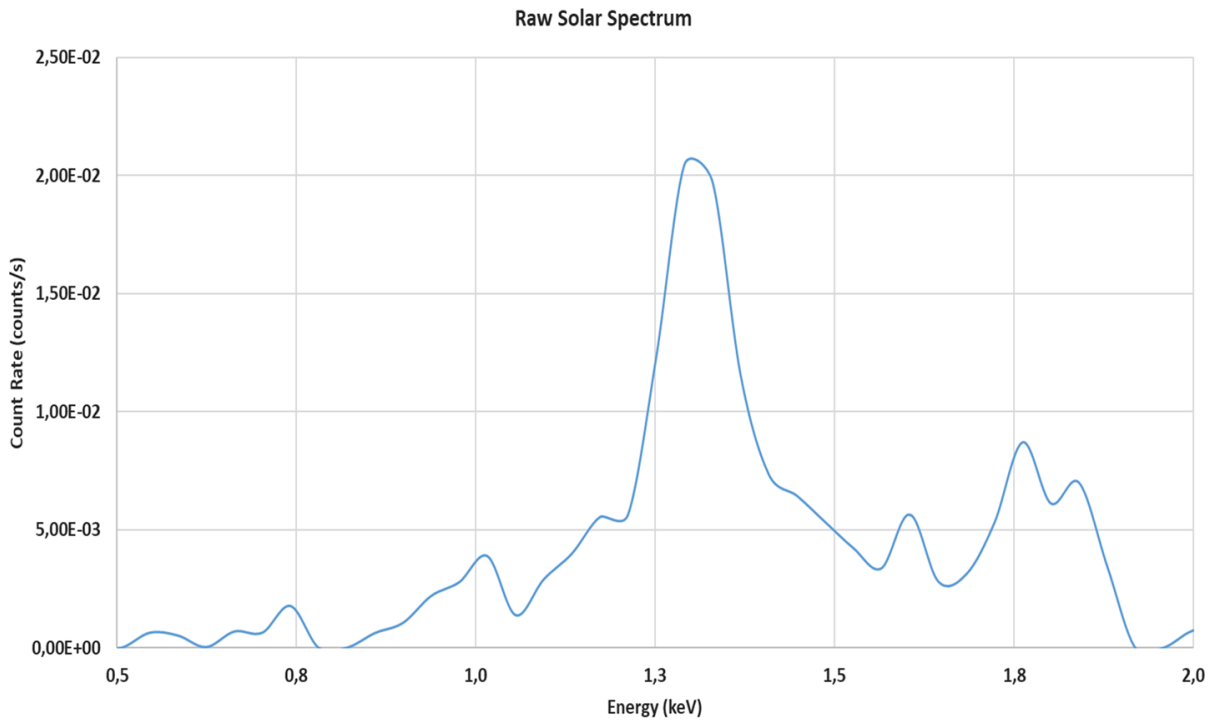

Fig. 24 Raw solar X-ray spectrum from $0.5 \mathrm{keV}$ to $2.0 \mathrm{keV}$. The contribution of solar electrons at above $20 \mathrm{keV}$ is not shown. The few negative count rate values due to statistical fluctuations of the signal have been removed

to the distance of $1 \mathrm{AU}$ is only $5 \cdot 10^{-9} \mathrm{~W} / \mathrm{m}^{2}$ (corresponding to X-ray flux at GOES A 0.5 level), which is significantly lower than the GOES flux A8 recorded in the public database. However, the lower limit of the energy bounds of GOES channels are only determined by 


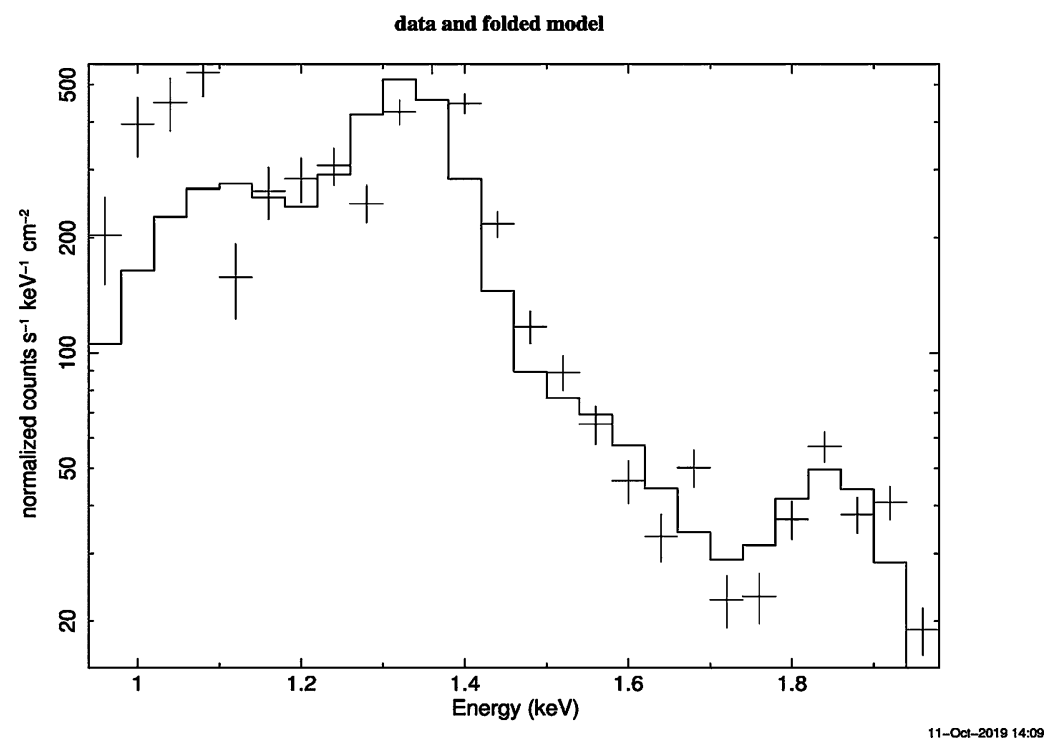

Fig. 25 Solar spectrum with fitted VAPEC model. The uncertainty below $1.2 \mathrm{keV}$ is due to very low quantum efficiency (Fig. 12) below $1.2 \mathrm{keV}$. The fit to the continuum above $1.2 \mathrm{keV}$ is relatively good despite the low number of raw counts. Two spectral lines are visible at $1.4 \mathrm{keV}$ and at $1.85 \mathrm{keV}$. These are the spectral lines of ionized $\mathrm{Mg}(1.4 \mathrm{keV})$ and $\mathrm{Si}(1,85 \mathrm{keV})$

Table 3 The model parameters of the VAPEC model. Only the increased elemental abundances of $\mathrm{Mg}$ and $\mathrm{Si}$ are shown here. The other elemental abundances were fixed at solar levels. "times solar" means, that the observed abundances of $\mathrm{Mg}$ and $\mathrm{Si}$ are shown here in the units of the known solar abundances of these elements

\begin{tabular}{ll}
\hline Parameter & Value \\
\hline Temperature & $0.231 \pm 0.012 \mathrm{keV}\left(2.7 \cdot 10^{6} \mathrm{~K}\right)$ \\
Mg abundance & $3.1 \pm 0.3$ times solar \\
Si abundance & $2.0 \pm 0.4$ times solar \\
Norm & $7.7 \cdot 10^{3} \pm 2.0 \cdot 10^{3}$ \\
\hline
\end{tabular}

the filter materials and thicknesses. GOES-15 detector has a $53 \mu \mathrm{m}$ Be entrance window, so the steepness of the cut-off towards lower energies is close to that of SIXS-X detectors, which have $50 \mu \mathrm{m}$ Be windows. Within the energy range 1-2 keV, the X-ray flux measured by SIXS is $8.4 \cdot 10^{-8} \mathrm{~W} / \mathrm{m}^{2}$, corresponding to X-ray flux at GOES A 8.4 level, which is in good agreement with the GOES measurement. This indicates that the GOES low energy channel measurements may, in fact, extend to considerably lower energies than the nominal $1.55 \mathrm{keV}$ limit. However, more observations with various $\mathrm{X}$-ray intensities are needed to confirm this.

This observation also shows that the instrument background, aside from the in-flight calibration signal, is very low and enables detection of extremely weak X-ray emission, such as that at 1.17 AU distance from the Sun on June 4th 2019. This makes scientific measurements with solar X-ray fluxes below the designed dynamic range of SIXS-X feasible by summing up the individual spectra as long as the conditions remain stable. It also gives 
an indication of good capability to provide high quality solar X-ray data for the analysis of MIXS data and independent scientific studies of the solar corona.

\subsection{Particle Detection System}

\subsubsection{Cosmic-Ray-Background Dominated Pulse Height Data}

During the measurement period after the launch of BepiColombo the Sun has been extremely quiet. The particle detection system, therefore, mainly observes background, which is due to very high energy cosmic-ray protons. Figure 26 shows all gathered pulse height data from the period of 13-23 June 2019 through Side 0 as a matrix of two 6-bit logarithmic pulse heights from the Side and the Core detector. Particles of different species and energies fall in different places of the matrix. The triangles give the proton track in ground calibrations in the accelerator campaigns. The diamonds give the same track but with the 11-bit energy loss signal in the side detector multiplied by 2 after offset subtraction, corresponding near the right-hand-side high-energy (left-hand-side low-energy) end of the track to the nominal track of protons at a $60^{\circ}\left(\sim 70^{\circ}\right)$ angle of incidence. The track produced by cosmic-ray particles between the triangles and diamonds is very broad, because the cosmicray energy spectrum is increasing as a function of energy and, thus, most of the detected protons have actually penetrated the collimator and have high angles of incidence to the detector. It is evident that very few protons in the nominal energy range of the coincidence measurement (4.3-30 MeV) have been observed, consistent with the quiet conditions and the form of the cosmic-ray energy spectrum.

Most of the cosmic-ray protons fall on a straight track above the line drawn from the point $(64,32)$ to the point $(32,0)$. These represent particles not stopping in the scintillator, but penetrating the whole instrument. The energies of these protons increase from $\sim 40 \mathrm{MeV}$ to the $\mathrm{GeV}$ range, when the two pulse heights representing energy losses in the detectors get smaller and smaller. The dark blue area in the matrix near the point $(34,4)$ is formed by minimally ionising particles, where cosmic-ray particles in a very broad range of energies (Lorentz factors $\sim 2-10$ ) will produce a very similar pair of signals in the detectors. The position of the particle on the track of penetrating protons has, therefore, information on the energy of the particle, allowing one to obtain some information of the cosmic-ray flux in integral energy channels. While the pulse-height sample gathered by SIXS-P (up to 21600 samples per day) is not enough to include all particle counts, it will allow us to gather a useful dataset for monitoring the long-term evolution of the cosmic-ray flux during the cruise. A detailed simulation study assessing the response of the detector to cosmic-ray protons has been initiated and will be a subject of a follow-up paper.

The offset of the core detector has not yet been tuned to match the lowest energy measurement, which leads to the gap in core detector pulses in Fig. 26 between 1 and 9 in the 6-bit logarithmic representation. Subtracting an additional offset of 10 units in the 11-bit linear pulse height would produce a matrix with counts in the range 1-9, as well, in the logarithmic 6-bit representation. However, to get an accurate value for the offset of the core, further in-flight calibrations with lower-energy electrons and protons are necessary. Such data will certainly be available from large solar energetic particle events during the cruise phase of the mission.

\subsubsection{Stream-Interaction-Region-Produced Low-Energy Proton Event in 16-23 June 2019}

In addition to cosmic-ray background, SIXS-P observed energetic $(\sim 1 \mathrm{MeV})$ protons from one solar-wind stream interaction region (SIR; Richardson 2018) on 16-23 June 2019. The 


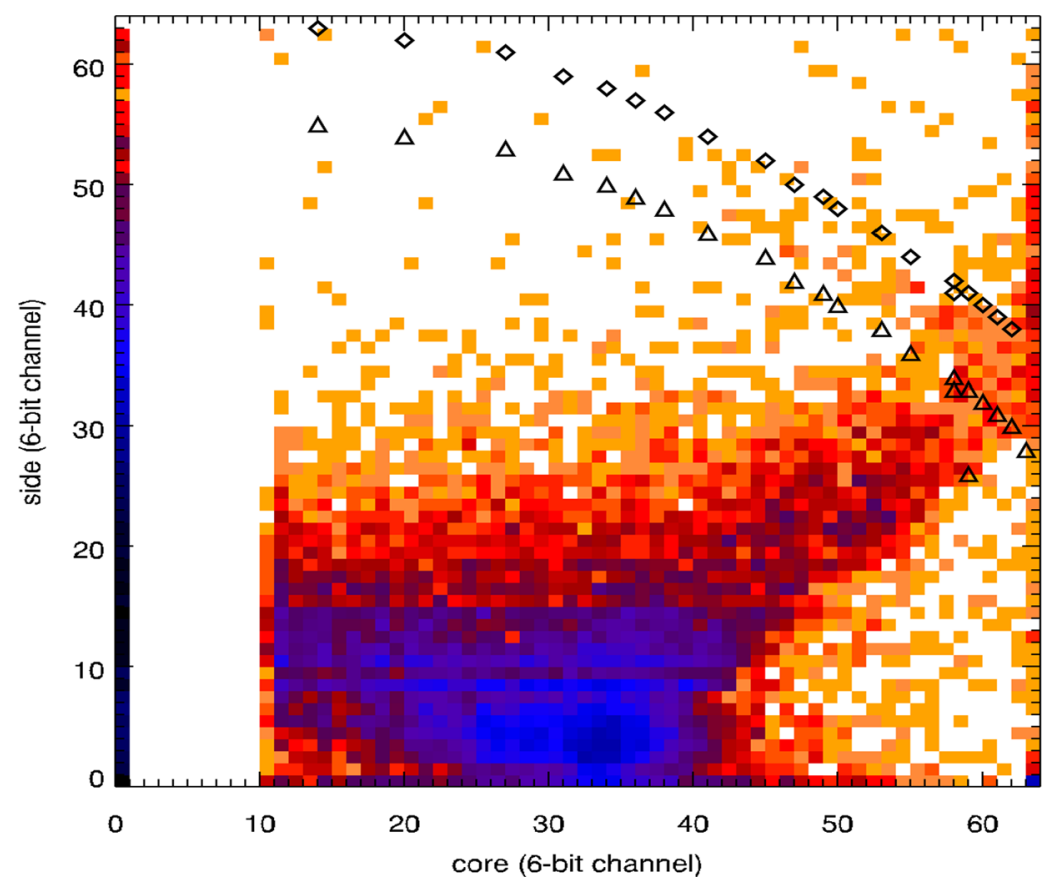

Fig. 26 In-flight response of SIXS particle detection system to quiet-time background from June 13-23, 2019 , detected by Side 0. The color (from orange to dark blue) represents counts in a bin of the 64 by 64 log-pulse-height matrix, the first column being those particles that do not trigger the core detector. The triangles are 4.5-55 MeV protons from the ground calibration campaign at $0^{\circ}$ angle of incidence. The diamonds are the same with the original 11-bit linear pulse height multiplied by a factor of 2, showing the track of protons with very inclined angles incident from above. The region between the two tracks should be populated by protons at these energies

spectrum in this event is extremely soft and the intensities are very low, hardly showing at all in the total counting rate of the instrument. However, the proton flux channel at 1.1$2.8 \mathrm{MeV}$ shows an increase of about a factor of three above the cosmic-ray background during the peak of the event on 16 June 2019 (Fig. 27). It is clear that event is caused by an SIR: there is a complete lack of solar flare activity during the time period and there is also a simultaneous (recurrent) proton event observed in SOHO/ERNE (Torsti et al. 1995) with lower levels of proton intensities. The solar wind speed measured by Charge, Element and Isotope Analysis System (CELIAS) on-board SOHO (Hovestadt et al. 1995) also shows a stream structure with alternating very low and intermediate wind speeds (Fig. 28) and there are no indications of plasma and field related to interplanetary CMEs (e.g., Kilpua et al. 2017) measured by the near-Earth spacecraft.

SOHO, in halo orbit around L1, was closer to the Sun than MPO in June 2019 (see Fig. 29). The outward radial gradient of proton intensity is consistent with the source being outside both spacecraft. Note that the geometric factor at low energies in SIXS is somewhat lower than nominal in the cruise configuration, which we have modeled by estimating that $70 \%$ of the field-of-view is unobstructed and records flux, whereas the other $30 \%$ is obstructed and sees no low-energy flux at all. However, systematic errors in the SIXS response at low energies are non-negligible and should be estimated by a simulation including the spacecraft structures in cruise configuration. As that is still a work in progress, we cannot 


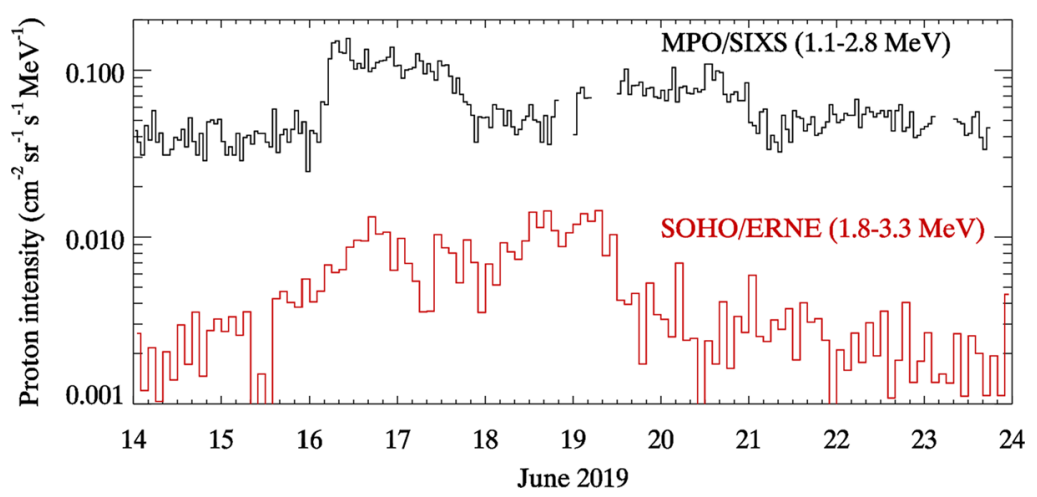

Fig. 27 Measurements by MPO/SIXS and SOHO/ERNE in 14-23 June 2019, showing increases in low-energy proton intensities related to an interaction region in the solar wind outside both spacecraft (see Fig. 29)

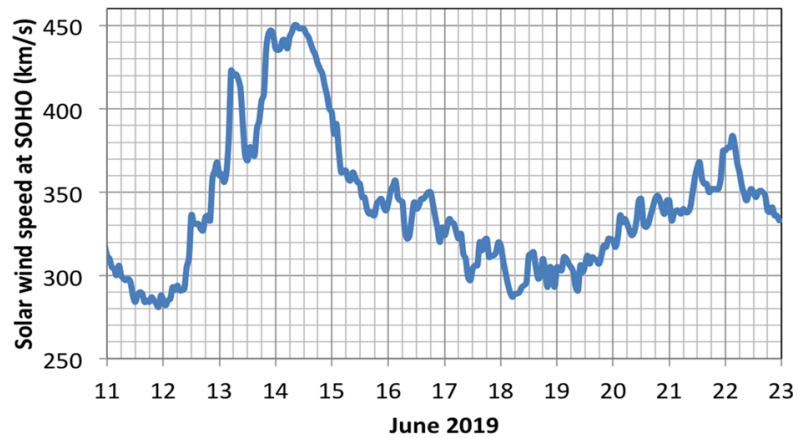

Fig. 28 Solar wind speed measured by SOHO/CELIAS in June 2019. The SIR related to the proton increase could have been formed by the steepening of the increase of the solar wind speed from its minimum value late on 11 June 2019 to the local maximum late in 13 June 2019 at about 1.8 AU distance from the Sun, as depicted in Fig. 29. Another SIR could have been formed further out by steepening of the speed increase from the minimum in 18 June 2019 to the maximum in 22 June 2019

yet compute reliable values for the radial gradient. However, the qualitative statement that they are positive in the SIR event can already be made, if we assume that the SIR is in steady state in the frame corotating with the Sun. The time lag of the two spacecraft being on the same streamline varies is on average about 30 hours so that SOHO precedes MPO. It seems that the main phase of the particle acceleration is related to a reverse mode shock that could form in the trailing edge of the first intermediate-speed solar wind stream observed by CELIAS in 14-18 June 2019. The later weak phases of the event can have contributions from the interplanetary shock structures bounding the second SIR forming further out due to the stream with peak speed at CELIAS on 22 June 2019. These proton fluxes, however, are very close to background levels both at MPO and SOHO.

The SIR proton event starts at MPO on 16 June, about a day after the connection to the SIR is established along the streamlines in the corotating frame. We estimate the location of the SIR by plotting the high- and low-speed solar wind streamlines from the location of SOHO and estimating where they would intersect. Since the energetic particles mainly travel along the actual magnetic field lines rather than the Parker field (which is parallel to the streamlines in the corotating frame), this delay is not inconsistent with the observations 


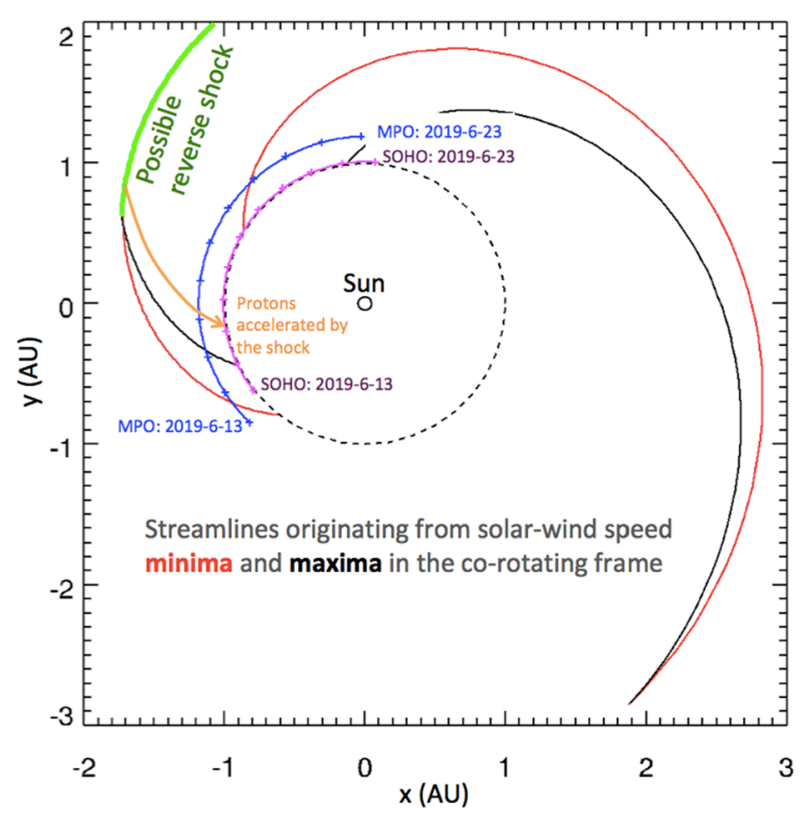

Fig. 29 Locations of MPO (blue line with crosses) and SOHO (purple line with crosses) in the ecliptic plane in 13-22 June 2019 in the frame corotating with the Sun. The dashed circle is at 1 AU distance from the Sun. The crosses on the spacecraft trajectories are the spacecraft positions at the beginning of each day of the period and the spacecraft are moving clockwise. The black and red lines correspond to streamlines in the rotating frame, calculated from the local maximum (on June 13 and 22) and minimum (on June 12 and 19) values, respectively, measured by the CELIAS instrument on-board SOHO (see Fig. 28). The green curve outlines a possible reverse-mode shock bounding the SIR causing the low-energy proton increase in MPO and $\mathrm{SOHO}$, as indicated by the orange arrow depicting protons propagating towards the Sun along the nominal Parker field, coinciding with the streamlines in the corotating frame

of energetic particles. The earlier increase in SOHO than in MPO is also consistent with the scenario. The main portion of the event seems to be observed when the spacecraft would be connected to the trailing edge of a stream interaction region driven by the stream with the peak in 14 June 2019 at SOHO.

The background level in SIXS is clearly higher (by a factor of 30) than in ERNE, which is natural as ERNE measurements are from a coincidence channel whereas SIXS measurements are from a single-detector channel. It is clear that SIXS can observe even small events like the SIR event of June 2019 with meaningful statistics despite its small geometric factor. This indicates that SIXS measurement will not only benefit the planetary research objectives at the orbit around Mercury but also the solar and heliospheric science that can be performed also during the cruise and in orbit around Mercury.

\section{Science Operations Plan for SIXS}

BepiColombo will have 7.2 years journey towards Mercury, During this time, it will travel through the inner heliosphere with varying heliospheric distances and longitudes, and there will be Earth, Venus and Mercury fly-bys. This phase is called the cruise phase. After the arrival in Mercury's orbit, there will be a maximum of 2 years of science operations, the 
science phase. The mission phases are described in detail in Benkhoff et al. (2020). The SIXS science operations plan includes operations in all phases of the mission.

\subsection{Cruise Phase}

Although BepiColombo's main target is Mercury, the observations during the cruise phase and flybys provide valuable information. Not all instruments can be active, but SIXS can do relevant science during this time as well.

\subsubsection{Planetary Fly-Bys}

In total there will be 9 planetary flybys during the cruise phase, one Earth flyby (APRIL 10, 2020), two Venus flybys (OCTOBER 15, 2020 AND AUGUST 11, 2021), and six Mercury flybys (OCTOBER 2, 2021; JUNE 23, 2022; JUNE 20, 2023; SEPTEMBER 5, 2024; DECEMBER 2, 2024 AND JANUARY 9, 2025) before BepiColombo enters the Mercury orbiting phase of the mission. For a detailed schedule of the fly-bys, see (Benkhoff et al. this issue).During the Earth flyby key science objectives for SIXS are observing Earth in X-rays (SIXS-X) and observing energetic particles when BepiColombo traverses through the Van Allen radiation belts. In addition, SIXS-X could possibly detect X-rays due to auroral activity at Earth, which also would be helpful for considering changes to detect aurora-kind phenomena at Mercury by SIXS. Earth flyby is also a useful phase for calibration between SIXS-P and the ERNE instrument onboard the SOHO spacecraft and SIXS-X background calibration and cross-calibration with the GOES spacecraft.

During the Venus fly-bys, SIXS can monitor energetic protons and electrons and SEP effects detection on atmospheric escape. If solar flare occurs during a flyby SIXS could provide the first direct measurement of SEPs in the Venus plasma environment and significant new information regarding SEPs. For example, relevant questions are

- How solar energetic particles (SEPs) drive atmospheric escape from Venus? (needs joint observations with other instruments from BepiColombo)

- How the properties of SEPs incident on the Venus atmosphere and in the induced magnetosphere differ from SEPs in interplanetary space?

- Is there planetary shadowing of SEPs?

During the Mercury fly-bys SIXS can already observe X-ray and SEPs from the Sun close at the Mercury's orbit and together with the other instruments of BepiColombo study their effects on Mercury's near-space environment.

There are no specific pointing requirements for flybys. Observations during flybys should start at least 24 hours before the flyby and last as long as the planetary bow shock is crossed.

\subsubsection{Cruise Science in Inner Heliosphere}

The majority of the time during the cruise phase BepiColombo is not in the vicinity of any planet, but will be travelling in interplanetary space at varying heliospheric distances.

During the cruise phase all measurements with SIXS-X are performed with detector 1, i.e., the one that can see the sky, and with specific attitude manouvre, also the Sun in its field-of-view. During the cruise phase, it is thus possible to scan the sky X-ray spectrum with SIXS-X. This will provide valuable new data for the determination of the distribution of X-ray emission from astrophysical sources and the sky X-ray background in an energy range and energy resolution relevant for large X-ray observatories such as XMM Newton. 
The relevant science questions to be addressed at this time with SIXS-X are

- What is the X-ray spectrum of the whole sky?

- How does the X-ray emission from astrophysical sources and sky X-ray background vary in time?

- What are the properties and dynamics of solar flares?

- How does the occurrence and properties of X-ray solar flares vary with solar activity

During the cruise phase SIXS-P will make measurements with the SCIENCE 1 mode. There are no attitude constraints. The relevant science questions to be addressed in the cruise phase outside flybys with SIXS-P can contribute are

- What are the mechanisms of energetic particle transport in the inner heliosphere?

- What are the acceleration mechanisms of solar energetic particles in the coronal shocks?

- How does energetic particle transport and acceleration vary with heliospheric distance in the inner heliosphere?

The cruise phase is expected to cover the minimum, rising and maximum phases of Solar Cycle 25, which is predicted to peak around 2024 with the strength similar or slightly stronger than for Solar Cycle 24 (e.g., Bhowmik and Nandy 2018). SIXS is thus expected to observe a large number of solar flares and coronal mass ejections (CMEs) and related particle acceleration events.

\subsection{Mercury Orbiting Phase/Science Phase}

The Mercury orbiting/science phase is the goal of BepiColombo mission. During this time SIXS will perform its actual task, i.e., measure the solar X-ray and particle input on Mercury's surface, simultaneously with observations of MIXS, but also independently. SIXS-X has one operational science mode (SCIENCE). SIXS-P has two science modes. The SCIENCE1 mode has a lower data resolution and is targeted for routine observations. The SCIENCE2 mode has additional higher data resolution delivery, and the high resolution data is stored for an onboard mass memory to wait for a selective downlink of high particle flux events.

Observations with SIXS-X are planned at all phases when the Sun is visible in the fieldof-view of any of the three X-ray detectors of SIXS. For SIXS-P the plan is to make observations during all orbital phases of Bepi/MPO. The only other operational constraint is the availability of power for operations, since both SIXS detection systems have very wide field-of-view, and can make scientifically useful and valuable observations independently of the $\mathrm{S} / \mathrm{C}$ attitude.

In the science phase, the SIXS particle detector system is operated in Science mode all the time. Periodical interruptions may be made for in-flight calibration and annealing as necessary. SIXS X-ray detectors can only be run one at a time. Their overlapping fields of view cover the section of sky that the Sun crosses when the MPO orbits Mercury with the $+Z$ axis pointing to the surface. The three SIXS-X detectors are run in sequence as the Sun crosses their respective fields of view. The sequence depends on the position of Mercury on its orbit around the Sun.

On the day side of the MPO orbit SIXS-X is run in science mode. In-flight calibrations and annealing are done on the night side. For SIXS-X, regular in-flight calibration is necessary for accurate spectral fitting and to keep track of the instrument health. The operation phases of SIXS are shown in Fig. 30. 
SIXS Operations during a Mercury orbit

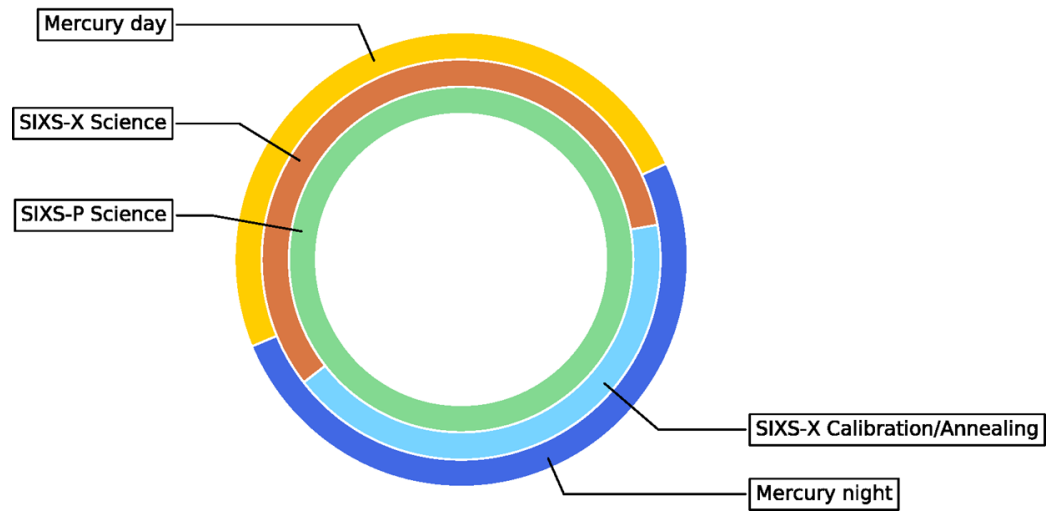

Fig. 30 SIXS regular operation cycles in Mercury's orbit

The Mercury orbiting phase is expected to coincide with maximum and early declining phase of Solar Cycle 25 (e.g., Bhowmik and Nandy 2018). During this time Sun leashes the most powerful solar flares and CMEs and Mercury is thus expected to be influenced by relatively strong energetic solar particle and X-ray fluxes.

Combined to cruise phase observations, particle and X-ray measurements of SIXS will provide a particularly long period of observations at varying solar cycle phases made in the inner heliosphere.

\section{Collaboration}

Joint measurements with MIXS are the main science objective of SIXS. SIXS measurements are also useful for SERENA and several other instruments onboard both the MPO the MMO. SIXS also benefits from combining the observations with the other instruments (Milillo et al. 2020). Simultaneous measurements by SIXS and other instruments of BepiColombo is beneficial for exploring many processes and phenomena at Mercury more comprehensively, including magnetospheric and exospheric dynamics, interactions with solar particles and radiation, and interplanetary dust. Such collaborative observations are also crucial for quantifying temporal and spatial dependencies.

SIXS-X will provide information of Sun major events associated with flares arriving at Mercury when Mio will be inside the magnetosphere, so not able to monitor the external conditions. In those configurations, SIXS together with BERM, monitoring the high energy radiation, are of crucial importance for the investigation of the Sun-planet interactions with the other instrument devoted to the Hermean environment like SERENA, MAG and the whole Mio instrumentation. During large SEP events, a significant portion of the highenergy particles will have direct access to the closed-field-line inner magnetosphere and can reach the planet. The circulation of these energetic particles in the magnetosphere and their interaction with the surface producing charged and neutral particle release can be investigated through joint observations of SIXS-P with MPO/MAG, SERENA, and PHEBUS and the Mio instruments.

Mariner-10 and MESSENGER detected energetic electrons (up to $200 \mathrm{keV}$ (Ho et al. 2012)) in the SIXS lower energy range. They are signature of substorm activity in the small 
magnetosphere of Mercury. Joint observations of SIXS with the Mio/MPPE-HEP instrument will contribute in tracing the electron trajectories and energisation during these events. During cruise phase there is an added value from simultaneous measurements with other particle instruments and magnetometers on BepiColombo.

The other missions that can be very valuable for SIXS are Solar Orbiter, Parker Solar Probe, STEREO and near-Earth spacecraft monitoring the Sun, X-rays and energetic particles. These missions will also provide collaboration opportunities during the cruise phase. Especially combining SIXS-P observations of solar energetic particle events with data near 1 AU (near-Earth and L1 spacecraft as well as STEREO-A) and data obtained closer to the Sun by the Parker Solar Probe and Solar Orbiter missions will provide an important addition to the analysis of coronal and interplanetary particle acceleration and transport.

Also, the near future Space Weather missions of ESA (Lagrange 5 mission) and NOAA/NASA (SWFO/Lagrange 1) are beneficial for improving the science output of SIXS, and will give good reference and cross-calibration possibilities, since they will have a closely similar, but more advanced X-ray Flux Monitors, which have the same heritage as SIXS. The XFM instruments are currently being designed, and will be procured to ESA by an industrial consortium in Finland for the ESA S2P (Space Safety Programme) within the next five years. The operations of at least the SWFO mission will be partly aligning with the time of BepiColombo operations during the science phase.

\section{Conclusions}

ESA's and JAXA's joint mission BepiColombo was successfully launched towards Mercury on October 19, 2018. One of the instruments carried on-board the Mercury Planetary Orbiter, called "Bepi", is the Solar Intensity X-ray and particle Spectrometer (SIXS). SIXS measures X-rays (SIXS-X) and energetic electrons and protons (SIXS-P) that are emitted primarily by the Sun. This impact causes X-ray emission of Mercury's surface. The X-ray emission contains information on the elemental composition and scattering properties of the Hermean surface. The X-ray emission may also include a component from the planet's weak magnetosphere via charged particle precipitation, in principle similarly as the X-rays above the polar regions of the Earth grow stronger during increased particle fluxes connected to space weather. Using simultaneous measurements of SIXS and MIXS, and a proper physical model, it is possible to derive the elemental composition and obtain information on the other phenomena on Mercury's surface. Requirements by MIXS thus constrain the spectral and time resolution, and energy ranges of SIXS X-ray and particle measurements.

SIXS data is also valuable for independent science, and is useful when combined with data from other instruments onboard BepiColombo. One key aspect for independent science objectives are long time series of high-quality observations X-rays and particles in the inner heliosphere, where such measurements have not been available before. SIXS is expected to yield new information on the dynamics and properties of solar eruptions, especially flares, and the mechanisms of energetic particle acceleration and transport in the corona and the heliosphere, as well as the dynamical processes in the Hermean magnetosphere.

BepiColombo performs a seven-year cruise phase in the inner heliosphere before arriving at Mercurys's orbit. SIXS can make observations already in the cruise phase and several planetary flybys, since the instrument has an open viewing sector to the sky during the cruise. In particular, SIXS-P can observe energetic particles as well as cosmic ray background during the whole cruise phase and the planet flybys. SIXS-X can also make observations of the 
$\mathrm{X}$-ray emission of the planets and the sky, and in special circumstances also the X-rays from the Sun.

The design, calibration and testing of SIXS have benefitted from earlier similar instruments developed by the SIXS team in Finland. SIXS-X is a next generation version of XSM instruments that flew onboard Smart-1 and Chandrayaan-1 in the turn of the millenium. The SIXS-P system has even longer heritage, since it has been developed in Turku, where the ERNE instrument of SOHO was also developed about 10 years earlier.

Modelling, simulations, and testing in the laboratory have indicated that sufficient performance of SIXS can be maintained throughout the mission and that the instrument can make all planned observations and the sensitivity range is sufficient for capturing the whole range from the weakest to the strongest expected solar events.

Early data obtained in the commissioning and early cruise phase have demonstrated that the performance of the instrument is the same in space, and that all functionalities work nominally. While solar activity has been low, and has yielded only a limited data for inflight confirmation of the upper limits of performance, the early results show that SIXS can detect very weak X-ray emission, and has extremely low internal counter background. SIXS-X observed the Sun during a slew maneuver on June 4, 2019. The X-ray solar X-ray emission was very weak (corresponding to A7 level in the solar X-ray flux scale based on GOES Low channel measurements), which is below the nominal dynamical range of SIXS-X. The data were, however, analyzed by summing up a large number of accurately preprocessed and calibrated individual spectra using SIXS science data processing pipeline. The analysis showed that the X-ray counter background is very low and SIXS can observe the spectrum of the extremely weak X-ray emission from the Sun even at the distance about $1.2 \mathrm{AU}$ from the Sun. This opens possibilities for studies of the solar X-ray corona and its processes in very quiescent states. This may give new insights in the studies of e.g., X-ray bright points and very small flares, especially during the science phase of BepiColombo mission. Due to the absence of large SEP events, SIXS-P has observed mainly cosmic-ray proton background so far. However, also SIXS-P detected a weak event of accelerated electrons and protons by a stream interaction region, showing that also SIXS-P has high sensitivity and sufficiently low instrument noise. Further calibrations will be performed during the cruise phase, first time when BepiColombo crosses Van Allen radiation belts during the Earth flyby in the spring of 2020.

Solar activity is expected to increase during the next years, and the majority of the cruise phase and Mercury orbiting main science phase are expected to coincide with rising and maximum phases of the solar cycle. Thus, more frequent flares and powerful solar eruptions and SEP events are expected, which improves the chances to catch strong SEP events during the occasional cruise phase. Slow-fast stream interaction regions that can also effectively accelerate charged particles are, in turn, observed in all solar cycle phases. The possibilities for obtaining X-ray measurements of strong solar flares during cruise phase are limited severely by the fact, that the nominal $\mathrm{S} / \mathrm{C}$ attitude during cruise does not allow visibility of the Sun for SIXS-X, and suitable special attitude changes are possible very rarely. However, when BepiColombo arrives at its' final destination orbit of Mercury, the Sun should be active, and a lot of useful data including frequent measurements of intermediate-to-high Xray flux and medium-to-big flares are expected to accumulate, enabling a lot of valuable science data for Mercury's surface studies with SIXS \& MIXS, for studies of the hot solar corona and flares, and investigations of the effects of the Sun in Mercury's exosphere.

In summary, both pre-light test and first results in space during the cruise phase show that SIXS is preforming as expected. The first observations indicate that both X-ray and particle detector systems have very low internal counter background, which will extend the 
possibilities to measure weaker phenomena than expected without the penalty in the high end of the dynamical range. Thus, SIXS can be expected to perform its science objectives successfully, and meet the science goals. However, one must keep in mind, that there is long way to go.

Acknowledgements Open access funding provided by University of Helsinki including Helsinki University Central Hospital. We acknowledge the European Space Agency (ESA) for providing us the opportunity to provide contribution to the payload of BepiColombo mission. We wish to express our sincere gratefulness to Business Finland (former Tekes) for the full financial support of the main Finnish contributions in the Mercury Planetary Orbiter, which are the SIXS instrument, the common digital processing unit (DPU) for SIXS and MIXS, and the onboard software (SW) for SIXS and MIXS. Research by KM partially funded by the Academy of Finland project 325805. RV and EK acknowledge funding of the Finnish Centre of Excellence in Research of Sustainable Space (Academy of Finland grant numbers 312357, 312390). EK acknowledges the SolMAG project (ERC-COG 724391) funded by the European Research Council (ERC) in the framework of the Horizon 2020 Research and Innovation Programme and the Academy of Finland project SMASH (310445). Last but not least, we would also like to thank the whole BepiColombo team at ESA, and especially the project (now mission) scientist, Johannes Benkhoff, the European instrument teams, and the Japanese MMO/Mio team, for the great spirit, innovativeness, collaborative mindset, and enthusiasm that helped our team to reach the best performance, and has made the whole journey enjoyable to all of us.

Publisher's Note Springer Nature remains neutral with regard to jurisdictional claims in published maps and institutional affiliations.

Open Access This article is licensed under a Creative Commons Attribution 4.0 International License, which permits use, sharing, adaptation, distribution and reproduction in any medium or format, as long as you give appropriate credit to the original author(s) and the source, provide a link to the Creative Commons licence, and indicate if changes were made. The images or other third party material in this article are included in the article's Creative Commons licence, unless indicated otherwise in a credit line to the material. If material is not included in the article's Creative Commons licence and your intended use is not permitted by statutory regulation or exceeds the permitted use, you will need to obtain permission directly from the copyright holder. To view a copy of this licence, visit http://creativecommons.org/licenses/by/4.0/.

\section{References}

L. Alha, J. Huovelin, T. Hackman, H. Andersson, C.J. Howe, E. Esko, M. Väänänen, The in-flight performance of the X-ray Solar monitor (XSM) on-board SMART-1. Nucl. Instrum. Methods Phys. Res., Sect. A 596, 317-326 (2008). https://doi.org/10.1016/j.nima.2008.08.138

L. Alha, J. Huovelin, K. Nygård, H. Andersson, E. Esko, C.J. Howe, B.J. Kellett, S. Narendranath, B.J. Maddison, I.A. Crawford, M. Grande, P. Sreekumar, Ground calibration of the Chandrayaan1 X-ray Solar Monitor (XSM). Nucl. Instrum. Methods Phys. Res., Sect. A 607, 544-553 (2009). https://doi.org/10.1016/j.nima.2009.06.049

M.J. Aschwanden, S.L. Freeland, Automated solar flare statistics in soft X-rays over 37 years of GOES observations: the invariance of self-organised criticality during three solar cycles. Astrophys. J. 754, 112 (2012). https://doi.org/10.1088/0004-637X/754/2/112

J. Benkhoff et al., this issue (2020)

B. Bhowmik, D. Nandy, Prediction of the strength and timing of sunspot cycle 25 reveal decadal-scale space environmental conditions. Nat. Commun. 9, 5209 (2018)

E. Bunce et al., this issue (2020)

U. Feldman, K.G. Widing, Elemental abundances in the solar upper atmosphere derived by spectroscopic means. Space Sci. Rev. 107, 665-720 (2003). https://doi.org/10.1023/A:1026103726147

G.W. Fraser, J.D. Carpenter, D.A. Rothery et al., The Mercury Imaging X-ray Spectrometer (MIXS) on BepiColombo. Planet. Space Sci. 58(1-2), 79-95 (2010)

J. Gieseler, P. Oleynik, H. Hietala, R. Vainio, H.-P. Hedman, J. Peltonen, A. Punkkinen, R. Punkkinen, T. Säntti, E. Hæggström, J. Praks, P. Niemelä, B. Riwanto, N. Jovanovic, M.R. Mughal, Radiation RADMON aboard Aalto-1 CubeSat: first results. Adv. Space Res. (2019). https://doi.org/10.1016/j.asr.2019.11.023

A. Harjunmaa, Auringon roihu- ja hiukkaspurkausten aiheuttaman röntgenemission käyttö Merkuriuksen pintakoostumuksen määrittämisessä. MSc thesis, University of Helsinki (2004). (In Finnish) 
E. Heino, Calibration and simulations of SIXS-P's response to energetic particles. M.Sc. Thesis, University of Turku (2016). http://urn.fi/URN:NBN:fi-fe2016080822628

Heyner et al., this issue (2020)

D. Hovestadt, M. Hilchenbach, A. Bürgi et al., CELIAS - charge, element and isotope analysis system for SOHO. Sol. Phys. 162, 441 (1995). https://doi.org/10.1007/BF00733436

J. Huovelin, L. Alha, H. Andersson, T. Andersson, R. Browning, D. Drummond, B. Foing, M. Grande, K. Hämäläinen, J. Laukkanen, V. Lämsä, K. Muinonen, M. Murray, S. Nenonen, A. Salminen, H. Sipilä, I. Taylor, O. Vilhu, N. Waltham, M. Lopez-Jorkama, Planet. Space Sci. 50, 1345-1353 (2002)

J. Huovelin, R. Vainio, H. Andersson et al., Solar Intensity X-ray and particle Spectrometer (SIXS). Planet. Space Sci. 58(1-2), 96-107 (2010). https://doi.org/10.1016/j.pss.2008.11.007

E.K.J. Kilpua, H.E.J. Koskinen, T.I. Pulkkinen, Coronal mass ejections and their sheath regions in interplanetary space. Living Rev. Sol. Phys. 14, 1 (2017). https://doi.org/10.1007/s41116-017-0009-6

J.M. Laming, The FIP and inverse FIP effects in solar and stellar coronae. Living Rev. Sol. Phys. 12, 2 (2015). https://doi.org/10.1007/lrsp-2015-2

A. Lehtolainen, J. Huovelin, L. Alha, T. Tikkanen, Estimates of in-flight calibration source activities for the SIXS X-ray detectors on board BepiColombo. Nucl. Instrum. Methods Phys. Res., Sect. A 636(1), 48-60 (2011). https://doi.org/10.1016/j.nima.2011.01.026

A. Lehtolainen, L. Alha, J. Huovelin, R. Moissl, S. Korpela, H. Andersson, K. Kuparinen, Ground calibrations of the Solar Intensity X-ray Spectrometer (SIXS) on board BepiColombo. Nucl. Instrum. Methods Phys. Res., Sect. A 735, 496-511 (2014). https://doi.org/10.1016/j.nima.2013.10.006

R.P. Lin, B.R. Dennis, G.J. Hurford et al., The Reuven Ramaty High-Energy Solar Spectroscopic Imager (RHESSI). Sol. Phys. 210, 3 (2002). https://doi.org/10.1023/A:1022428818870

Milillo et al., this issue (2020). https://doi.org/10.1007/s11214-020-00712-8

Moissl et al., this issue (2020)

Murakami et al., this issue (2020)

J. Näränen, H. Parviainen, K. Muinonen, K. Nygård, M. Peura, J. Carpenter, Icarus 198, $408-419$ (2008)

J. Näränen, J. Carpenter, H. Parviainen, K. Muinonen, G. Fraser, M. Peura, A. Kallonen, Adv. Space Res. 44, 313-322 (2009)

S. Narendranath, P. Sreekumar, L. Alha, K. Sankarasubramanian, J.Huovelin.P.S. Athiray, Elemental abundances in the solar corona as measured by the X-ray solar monitor onboard Chandrayaan-1. Sol. Phys. 289(5), 1585-1595 (2014)

S. Narendranath, P. Sreekumar, S. Panini, N.S. Pillai, K. Sankarasubramanian, J. Huovelin, Coronal elemental abundances: New results from soft X-ray spectroscopy of the Sun. Astrophys. J. (2020, submitted)

P. Oleynik, R. Vainio, A. Punkkinen, O. Dudnik, J. Gieseler, H.-P. Hedman, H. Hietala, E. Hæggström, P. Niemelä, J. Peltonen, J. Praks, R. Punkkinen, T. Säntti, E. Valtonen, Calibration of RADMON radiation monitor onboard Aalto-1 CubeSat. Adv. Space Res. (2019). https://doi.org/10.1016/j.asr.2019.11.020

Orsini et al., this issue (2020)

H. Parviainen, J. Näränen, K. Muinonen, J. Quant. Spectrosc. Radiat. Transf. 112, 1907-1918 (2011)

Quémerais et al., this issue (2020)

I.G. Richardson, Solar wind stream interaction regions throughout the heliosphere. Living Rev. Sol. Phys. 15, 1 (2018). https://doi.org/10.1007/s41116-017-0011-z

Rothery et al., this issue (2020)

Saito et al., this issue (2020)

J.P. Santos, F. Parente, Y.-K. Kim, Cross sections for K-shell ionization of atoms by electron impact. J. Phys. B 36, 4211-4224 (2003)

K. Sera, K. Ishii, K. Kamiya, A. Kuwako, S. Morita, K-shell ionization of Al and Cu for 0.5-40-MeV-proton bombardment. Phys. Rev. A 21, 1412-1418 (1980)

T.C. Sorensen, T.P. Armstrong, A. Gandhi Pavanasam, S. Taherion, Galileo energetic particle detector observations of the spatial distributions and energy spectra of $>1$ and $>11 \mathrm{MeV}$ electrons in the 10 $40 \mathrm{RJ}$ region of the jovian magnetosphere. Icarus 178(2), 395-405 (2005). https://doi.org/10.1016/ j.icarus.2005.07.006

R.D. Starr, D. Schriver, L.R. Nittler, S.Z. Weider, P.K. Byrne, G.C. Ho, E.A. Rhodes, C.E. Schlemm II., S.C. Solomon, P.M. Trávníček, MESSENGER detection of electron-induced X-ray fluorescence from Mercury's surface. J. Geophys. Res. 117, E00L02 (2012). https://doi.org/10.1029/2012JE004118

J. Torsti, E. Valtonen, M. Lumme et al., Energetic particle experiment ERNE. Sol. Phys. 162, 505 (1995). https://doi.org/10.1007/BF00733438

M. Väänänen, L. Alha, J. Huovelin, Cross-calibration of SMART-1 XSM with GOES and RHESSI. Sol. Phys. 260(2), 479-488 (2009) 